\title{
OPTIMAL PORTFOLIO SELECTION WITH CONSUMPTION AND NONLINEAR INTEGRO-DIFFERENTIAL EQUATIONS WITH GRADIENT CONSTRAINT: A VISCOSITY SOLUTION APPROACH
}

\author{
FRED ESPEN BENTH, KENNETH HVISTENDAHL KARLSEN, AND KRISTIN REIKVAM
}

\begin{abstract}
We study a problem of optimal consumption and portfolio selection in a market where the logreturns of the uncertain assets are not necessarily normally distributed. The natural models then involve pure-jump Lévy processes as driving noise instead of Brownian motion like in the Black and Scholes model. The state constrained optimization problem involves the notion of local substitution and is of singular type. The associated Hamilton-Jacobi-Bellman equation is a nonlinear first order integro-differential equation subject to gradient and state constraints. We characterize the value function of the singular stochastic control problem as the unique constrained viscosity solution of the associated Hamilton-Jacobi-Bellman equation. This characterization is obtained in two main steps. First, we prove that the value function is a constrained viscosity solution of an integro-differential variational inequality. Second, to ensure that the characterization of the value function is unique, we prove a new comparison (uniqueness) result for the state constraint problem for a class of integro-differential variational inequalities. In the case of HARA utility, it is possible to determine an explicit solution of our portfolio-consumption problem. This is, however, the topic of a companion paper [7].
\end{abstract}

\section{INTRODUCTION}

We consider a model of optimal consumption and portfolio selection which captures the notion of local substitution. This optimization problem was first suggested and studied in detail by Hindy and Huang [16] for diffusion processes using verification theorems. Later, Alvarez [1] studied the problem in a viscosity solution framework. A viscosity solution approach has also been pursued by Hindy, Huang, and Zhu [17] for a certain generalization of this problem. The main motivation for the present paper is to generalize the results by Hindy and Huang [16] and Alvarez [1] to statistically sound models for the asset price process.

An agent wants to divide her wealth between an uncertain asset with price $S_{t}$ and a bond $B_{t}$ with interest rate $r$. She wants to allocate her wealth and at the same time consume in order to optimize the functional

$$
\mathrm{E}\left[\int_{0}^{\infty} e^{-\delta t} U\left(Y_{t}^{\pi, C}\right) d t\right]
$$

where $\pi=\pi_{t}$ denotes the fraction of wealth allocated in the uncertain investment and $C=C_{t}$ is the cumulative consumption at time $t$. This functional describes the agent's preferences over consumption patterns. The agent's utility is described by $U$, discounted by the rate $\delta$. The special feature of this problem introduced by Hindy and Huang [16] is the process $Y_{t}$ modeling the average past consumption. This process will be derived from the total consumption up to time $t$ and a certain weighting factor (see equation (2.7)). This model says that the agent derives satisfaction from past consumption. In addition, the control problem incorporates the idea of

Date: April 6, 2000.

Key words: Portfolio choice, intertemporal utility, stochastic control, singular control, dynamic programming, integro-differential variational inequality, state constraint problem, viscosity solution, comparison result.

JEL classification (1991): G11, C61, D91.

Mathematics Subject Classification (1991): 45K05, 49L20, 49L25, 93E20.

Acknowledgements: The research of F. E. Benth has been supported by MaPhySto-Centre for Mathematical Physics and Stochastics. MaPhySto is funded by a grant from the Danish National Research Foundation. The research of K. Reikvam has been supported by the Norwegian Research Council under grant NFR 118868/410. We would like to thank Said Elghanjaoui, Nils Christian Framstad, and Bernt $\varnothing$ ksendal for valuable discussions. 
local substitution which says that consumption at nearby dates are almost perfect substitutes. Advancing or delaying consumption has little effect on the consumer's satisfaction. With this model of satisfaction, optimal consumption was shown by [16] to be periodic in the sense of a local time on a boundary. Every time the wealth process hits a boundary, consumption takes place. We have chosen to consider the case of an agent with infinite investment horizon.

The standard model for stock prices in the Black and Scholes world is the geometric Brownian motion

$$
S_{t}=S_{0} e^{\mu t+\sigma W_{t}}
$$

where $\mu$ is the expected log-return and $\sigma$ the volatility. This model imposes a normal distribution on the logreturns of an observed stock price. Empirical work by Eberlein and Keller [14] and Rydberg [30] shows that the normal distribution poorly fits the logreturn data. Among other: things, the data have heavy tails. They suggest modeling logreturns by generalized hyperbolic distributions, which are shown to fit data extremely well. Barndorff-Nielsen [6] introduces the normal inverse Gaussian distribution, which is thoroughly studied on financial time series by Rydberg [30]. Eberlein and Keller [14] use the hyperbolic distribution. The model for stock prices becomes

$$
S_{t}=S_{0} e^{L_{t}},
$$

where $L_{t}$ is a Lévy process and $L_{1}$ is distributed according to a normal inverse Gaussian law in $[6,30]$ and a hyperbolic law in [14]. It is worth noticing that in both cases $L_{t}$ will be a pure-jump Lévy process, i.e., it does not have any Brownian motion part in its Lévy-Khintchine representation. Thus the generator of $S_{t}$ will have no second order term and our control problem - as will be explained later - will be a first order integro-differential variational inequality. We shall assume here that the stock price is driven by a general pure-jump Lévy process $L_{t}$.

By the Bellman principle we can associate a Hamilton-Jacobi-Bellman equation (variational inequality) to our optimization problem. This equation is set in an unbounded domain and consists of a nonlinear first order integro-differential equation subject to a gradient constraint, a so-called integro-differential variational inequality (see Section 2). Since we allow for consumption processes which are not necessarily absolutely continuous with respect to the Lebesgue measure, we have a so-called singular control problem. These problems give rise to a gradient constraint in the Hamilton-Jacobi-Bellman equation, see, e.g., Fleming and Soner [15]. In our general set-up, it is natural to consider the variational problem in the framework of viscosity solutions, as done by Alvarez [1] for the geometric Brownian motion case. We recall that the notion of viscosity solutions was introduced by Crandall and Lions [11] for first order equations and by Lions [26, 27] for second order equations. The notion of viscosity solutions for integro-differential equations was later pursued by Soner $[34,35]$ and Sayah $[31,32]$ for certain problems involving a first order local operator, and by Alvarez and Tourin [2] and Pham [29] for problems involving a second order: local operator. For control problems and their associated Hamilton-Jacobi-Bellman equations, this weak solution concept has proven to be extremely useful due to the fact that it allows merely continuous functions to be solutions of fully nonlinear second order partial differential equations. We refer to the user's guide of Crandall, Ishii and Lions [10], the lecture notes in [4], and the books $[3,5,15]$ for an overview of the theory of viscosity solutions and its applications.

For our problem, we need to consider constrained viscosity solutions since we are not allowed to consume more than the present wealth, e.g., the control cannot push the wealth process into the negative real line. The notion of constrained viscosity solutions was first introduced by Soner $[33,34]$ and later Capuzzo-Dolcetta and Lions [13] for first order equations, see also Lasry and Lions [24], Lions and Ishii [20], and Katsoulakis [23] for second order equations. In the present paper, we first prove that the value function of our control problem is a constrained viscosity solution of the associated integro-differential variational inequality (see Section 4). As observed by Lions (see, e.g., [27]), the general fact that value functions of control problems can be characterized as viscosity solutions of certain partial differential equations is a direct consequence of the dynamic programming principle. For singular control problems, however, the classical approach of Lions fails because the state process may jump due to the singular control and thus it needs not stay in a small ball for small $t$. This problem has usually been circumvented by either relying on the 
existence of an optimal control (see, e.g., $[12,17]$ ) or by establishing appropriate estimates for the state process (see, e.g., [15]). In [1], Alvarez presented a more direct argument showing that the value function of the singular control problem in [16] is a viscosity solution of the associated variational inequality. We adopt his argument to our singular control problem (where also the stock price process can jump) and its associated integro-differential variational inequality.

Our second result is a comparison principle for the state constraint problem for a class of integro-differential variational inequalities. This result ensures that the value function is the only solution of our problem, see Section 4. The first comparison principles (uniqueness results) for viscosity solutions were given by Crandall and Lions [11] (see also Crandall, Evans, and Lions [9]) for first order equations. Concerning the uniqueness theory for second order equations, important contributions are due to Jensen [21], Jensen, Lions, and Souganidis [25], Lions and Souganidis [28], Ishii [19], Jensen [22], and Ishii and Lions [20]. We refer to the user's guide [10], the lecture notes of Crandall [8], and the books [3, 5, 15] for an up-to-date overview of the uniqueness machinery for viscosity solutions.

Following the ideas set forth by the general uniqueness theory for viscosity solutions, comparison principles for integro-differential equations were obtained by Soner [34, 35], Sayah [31, 32], Alvarez and Tourin [2], and Pham [29]. Under some assumptions, uniqueness results in the class of bounded uniformly continuous (semiconcave) functions were obtained in [35], see also [34]. The main result of [31] is a comparison theorem between bounded uniformly continuous subsolutions and supersolutions. In [32], this result is extended first to semicontinuous and then to unbounded suband supersolutions. In [2], the authors consider nonlinear integro-differential equations of parabolic type and obtain a comparison principle for semicontinuous, bounded and unbounded sub- and supersolutions. In [29], a comparison principle is proved for unbounded sub- and supersolutions of an integro-differential quasi-variational inequality associated with the optimal stopping time problem in a finite horizon of a controlled jump-diffusion process.

We consider here a class of integro-differential variational inequalities for which the comparison results in the literature do not (directly) apply. We prove for this class of variational inequalities a comparison theorem between unbounded continuous subsolutions and supersolutions. Inspired by Ishii and Lions [20] in their treatment of general boundary value problems, we handle the gradient constraint by producing strict supersolutions that are close to the supersolution in question. A similar approach has also been used in, e.g., [12] for a singular stochastic control problem (without an integral operator), see also [1]. To handle the state constraint we adapt the proof of Soner $[33,34]$, which here consists in building a test function so that the minimum associated with the supersolution cannot be on the boundary. When dealing with unbounded domains, it is well known that one has to specify the asymptotic behaviour of the functions being compared. However, due to the choice of a strict supersolution, it is sufficient to restrict our attention to a bounded domain when proving the comparison principle. This was also done in [1].

Let us also mention that if we specialize to a utility function of HARA type, it is possible to construct an explicit solution of the portfolio-consumption problem studied in the present paper. This is the topic of our companion paper [7]. In that paper, we consider a more general model for the stock price which takes into account a Brownian component as well. In this case, the associated Hamilton-Jacobi-Bellman equation is a second order integro-differential variational inequality. In [7], we characterize the value function as the unique constrained viscosity solution of this equation.

Finally, for an overview of applications of viscosity solutions in mathematical finance, we refer to the lecture notes by Soner [36] and the references therein.

\section{Formulation of the PRoblem and the Main Result}

Let $(\Omega, \mathcal{P}, \mathcal{F})$ be a probability space and $\left(\mathcal{F}_{t}\right)$ a given filtration satisfying the usual assumptions. We consider a financial market consisting of a stock and a bond. Assume that the value of the stock follows the stochastic process

$$
S_{t}=S_{0} e^{L_{t}}
$$


where $L_{t}$ is a pure-jump Lévy process with Lévy-Khintchine decomposition

$$
L_{t}=\mu t+\int_{0}^{t} \int_{|z|<1} z \tilde{N}(d s, d z)+\int_{0}^{t} \int_{|z| \geq 1} z N(d s, d z) .
$$

Here $\mu$ is a constant, $N(d t, d z)$ is Poisson random measure on $\mathbb{R}_{+} \times \mathbb{R}$ with intensity measure $d t \times \nu(d z), \nu(d z)$ is a $\sigma$-finite Borel measure on $\mathbb{R} \backslash\{0\}$ with the property

$$
\int_{\mathbb{R} \backslash\{0\}} \min \left(1, z^{2}\right) \nu(d z)<\infty,
$$

and $\tilde{N}(d t, d z)=N(d t, d z)-d t \times \nu(d z)$ is the compensated Poisson random measure. The measure $\nu(d z)$ is called the Lévy measure. We choose to work with the unique càdlàg version of $L_{t}$ and denote this also by $L_{t}$. By Itô's formula (see, e.g., [18]) we obtain the differential form of $S_{t}$ :

$$
\begin{aligned}
d S_{t}=( & \left.+\int_{|z|<1}\left(e^{z}-1-z\right) \nu(d z)\right) S_{t} d t \\
& +S_{t-} \int_{|z|<1}\left(e^{z}-1\right) \tilde{N}(d t, d z)+S_{t-} \int_{|z| \geq 1}\left(e^{z}-1\right) N(d t, d z) .
\end{aligned}
$$

For this differential form to be well defined, we need to impose the following additional integrability condition on the Lévy measure:

$$
\int_{|z| \geq 1}\left|e^{z}-1\right| \nu(d z)<\infty
$$

Note that condition (2.4) is effective only when $z \geq 1$ due to (2.2), and says essentially that $e^{z}$ is $\nu(d z)$ - integrable on $\{z \geq 1\}$. Under condition (2.4), we can rewrite the differential form of $S_{t}$ as

$$
d S_{t}=\left(\mu+\int_{\mathbb{R} \backslash\{0\}}\left(e^{z}-1-z 1_{|z|<1}\right) \nu(d z)\right) S_{t} d t+S_{t-} \int_{\mathbb{R} \backslash\{0\}}\left(e^{z}-1\right) \tilde{N}(d t, d z) .
$$

Note that under condition (2.4), $\int_{0}^{t} \mathrm{E}\left[S_{s}\right] d s<\infty$.

We let the bond have dynamics

$$
d B_{t}=r B_{t} d t,
$$

where $r>0$ is the interest rate. Assume furthermore that $r<\hat{\mu}$, where we have introduced the short-hand notation

$$
\hat{\mu}=\mu+\int_{\mathbb{R} \backslash\{0\}}\left(e^{z}-1-z 1_{|z|<1}\right) \nu(d z) .
$$

Here, $r<\hat{\mu}$ means that the expected return of the stock is higher than the return of the bond. In (2.6), note that $e^{z}-1-z \geq 0$ for all $z \in \mathbb{R}$. Consider an investor who wants to put her money in the stock and the bond so as to maximize her utility. Let $\pi_{t} \in[0,1]$ be the fraction of her wealth invested in the stock at time $t$, and assume that there are no transaction costs in the market.

If we denote her cumulative consumption up to time $t$ by $C_{t}$, we have the wealth process $X_{t}^{\pi, C}$ given as

$$
X_{t}^{\pi, C}=x-C_{t}+\int_{0}^{t}\left(r+(\hat{\mu}-r) \pi_{s}\right) X_{s}^{\pi, C} d s+\int_{0}^{t} \pi_{s-} X_{s-}^{\pi, C} \int_{\mathbb{R} \backslash\{0\}}\left(e^{z}-1\right) \tilde{N}(d s, d z),
$$

where $x$ is the initial wealth. To incorporate the idea of local substitution, Hindy and Huang [16] introduce the process $Y_{t}^{\pi, C}$ modeling the average past consumption. The process has dynamics

$$
Y_{t}^{\pi, C}=y e^{-\beta t}+\beta e^{-\beta t} \int_{[0, t]} e^{\beta s} d C_{s},
$$

where $y>0$ and $\beta$ is a positive weighting factor. We shall frequently use the notation $Y_{t}$ for $Y_{t}^{\pi, C}$ and $X_{t}$ for $X_{t}^{\pi, C}$. The integral is interpreted pathwise in a Lebesgue-Stieltjes sense. The differential form of $Y_{t}$ is

$$
d Y_{t}=-\beta Y_{t} d t+\beta d C_{t} .
$$


The objective of the investor is to find an allocation process $\pi_{t}^{*}$ and a consumption pattern $C_{t}^{*}$ which optimize the expected discounted utility over an investment horizon. We shall here focus on an investor with an infinite investment horizon. We define the value function as

$$
V(x, y)=\sup _{\pi, C \in \mathcal{A}_{x, y}} \mathrm{E}\left[\int_{0}^{\infty} e^{-\delta t} U\left(Y_{t}^{\pi, C}\right) d t\right]
$$

where $\delta>0$ is the discount factor and $\mathcal{A}_{x, y}$ is a set of admissible controls. Let

$$
\mathcal{D}=\left\{(x, y) \in \mathbb{R}^{2}: x>0, y>0\right\}
$$

We say that a pair of controls is admissible for $x, y \in \overline{\mathcal{D}}$ and write $\pi, C \in \mathcal{A}_{x, y}$ if:

$\left(c_{i}\right) C_{t}$ is an adapted process that is right continuous with left-hand limits (càdlàg), nondecreasing, with initial value $C_{0-}=0$ (to allow an initial jump when $C_{0}>0$ ), and satisfies $\mathrm{E}\left[C_{t}\right]<\infty$ for all $t \geq 0$.

$\left(c_{i i}\right) \pi_{t}$ is an adapted càdlàg process with values in $[0,1]$.

(ciii) $X_{t}^{\pi, C} \geq 0, Y_{t}^{\pi, C} \geq 0$ almost everywhere for all $t \geq 0$.

Note that condition $\left(c_{i i i}\right)$ introduces a state space constraint into our control problem. The utility function $U:[0, \infty) \rightarrow[0, \infty)$ is assumed to have the following properties:

$\left(u_{i}\right) \quad U \in C([0, \infty))$ is nondecreasing and concave.

$\left(u_{i i}\right)$ There exist constants $K>0$ and $\gamma \in(0,1)$ such that $\delta>k(\gamma)$ and

$$
U(z) \leq K(1+z)^{\gamma}
$$

for all nonnegative $z$, where

$$
k(\gamma)=\max _{\pi \in[0,1]}\left[\gamma(r+(\hat{\mu}-r) \pi)+\int_{\mathbb{R} \backslash\{0\}}\left(\left(1+\pi\left(e^{z}-1\right)\right)^{\gamma}-1-\gamma \pi\left(e^{z}-1\right)\right) \nu(d z)\right] .
$$

By a Taylor expansion we see that the integral term of $k(\gamma)$ is well-defined in a neighbourhood of zero. The condition (2.4) ensures that the integral is finite outside this neighbourhood, which shows that (2.9) is finite for $\gamma \in(0,1]$. Recall that in the case of no integral operator in $(2.9), k(\gamma)$ maps $[0, \infty)$ onto $[0, \infty)$ with $k(0)=0$ and is increasing, see [1]. This is not the case when the integral operator is present. Then $k(\gamma):(0, \infty) \rightarrow \mathbb{R}$ can be negative as well as non-monotone. Moreover, one can easily check that the integral term in (2.9) is nonpositive. Let us also mention that condition $\left(u_{i i}\right)$ guarantees that the value function of the related Merton problem is welldefined, see [7].

In this paper we will assume that the dynamic programming principle holds: i.e., for any stopping time $\tau$ and $t \geq 0$,

$$
V(x, y)=\sup _{\pi, C \in \mathcal{A}_{x, y}} \mathrm{E}\left[\int_{0}^{t \wedge \tau} e^{-\delta s} U\left(Y_{s}^{\pi, C}\right) d s+e^{-\delta(t \wedge \tau)} V\left(X_{t \wedge \tau}^{\pi, C}, Y_{t \wedge \tau}^{\pi, C}\right)\right]
$$

where $a \wedge b=\min (a, b)$. This intuitive but important principle can be proved by using methods from, e.g., [37]. The Hamilton-Jacobi-Bellman equation of our optimization problem is a nonlinear first order integro-differential equation subject to a gradient constraint:

$$
\begin{aligned}
& \max \left\{\beta v_{y}-v_{x} ; U(y)-\delta v-\beta y v_{y}+\max _{\pi \in[0,1]}\left[(r+(\hat{\mu}-r) \pi) x v_{x}+\right.\right. \\
& \left.\left.\int_{\mathbb{R} \backslash\{0\}}\left(v\left(x+\pi x\left(e^{z}-1\right), y\right)-v(x, y)-\pi x v_{x}(x, y)\left(e^{z}-1\right)\right) \nu(d z)\right]\right\}=0 \text { in } \mathcal{D} .
\end{aligned}
$$

Note that $x+\pi x\left(e^{z}-1\right) \geq 0$ for all $x \geq 0$ and $z \in \mathbb{R}$. In Section 4 , we prove that if $v$ is $C^{2}$ and sublinearly growing, then (2.11) is well-defined. Moreover, if the value function $V$ defined in (2.8) satisfies these conditions, then by using Itô's formula one can easily prove that $V$ solves (2.11). 
Although (2.11) only contains first order derivatives, the requirement $V \in C^{2}$ comes from the fact that the Lévy measure $\nu(d z)$ is possibly singular in zero.

In many applications the value function is not necessarily smooth, or it can be very difficult to prove sufficient regularity. Therefore we introduce an appropriate concept of weak solutions, namely viscosity solutions. With this concept at hand, we are able to prove that the value function $V$ is the (only) solution of (2.11), even when it is not necessarily differentiable. However, if a viscosity solution is sufficiently regular, then, as is well known, it is a solution in the classical sense. The viscosity solution approach is by now a well established approach to control theory problems, see, e.g., the books [15, 3].

Our main result is the following theorem, which follows immediately from the results stated and proved in the Sections 3 and 4:

Theorem 2.1. The value function $V$ is the unique constrained viscosity solution of the integrodifferential variational inequality (2.11), i.e., $V$ is a subsolution of (2.11) in $\overline{\mathcal{D}}$ and a supersolution of $(2.11)$ in $\mathcal{D}$. The value function $V$ satisfies the growth condition

$$
0 \leq V(x, y) \leq K(1+x+y)^{\gamma}, \quad \forall x, y \in \overline{\mathcal{D}},
$$

and is uniformly continuous in $\overline{\mathcal{D}}$. Moreover, if for some $\alpha \in(0,1], \delta>k(\alpha)$ and $U \in C^{0, \alpha}([0, \infty))$, then $V \in C^{1, \alpha}(\overline{\mathcal{D}})$. Finally, if $\delta>k(1+\alpha)$ and $U \in C^{1, \alpha}([0, \infty))$, then $V \in C^{1, \alpha}(\overline{\mathcal{D}})$.

Before ending this section, we show that the normal inverse Gaussian Lévy process introduced by Barndorff-Nielsen [6] satisfies the condition in (2.4).

The normal inverse Gaussian distribution. First, recall from [6] and [30] that the normal inverse Gaussian distribution is a mean-variance mixture of a normal distribution and an inverse Gaussian with density

$$
\operatorname{nig}(x ; \alpha, \beta, \mu, \delta)=\frac{\alpha \delta}{\pi} \exp \left(\delta \sqrt{\alpha^{2}-\beta^{2}}+\beta(x-\mu)\right) \frac{K_{1}\left(\alpha \sqrt{\delta^{2}+(x-\mu)^{2}}\right)}{\sqrt{\delta^{2}+(x-\mu)^{2}}} .
$$

In (2.12), $K_{1}$ is the modified Bessel function of the third kind and index 1:

$$
K_{1}(y)=\frac{1}{2} \int_{0}^{\infty} \exp \left(-\frac{1}{2} y\left(x+x^{-1}\right)\right) d x, \quad \text { for } y>0,
$$

where $x \in \mathbb{R}, \mu \in \mathbb{R}, \delta>0$, and $0 \leq|\beta| \leq \alpha$. The parameters have the following meaning: $\alpha$ is the steepness of the distribution, $\beta$ the asymmetry, $\mu$ the location and $\delta$ the scale. ${ }^{1}$ If $\beta=0$ then the distribution is symmetric. The Lévy-Khintchine representation for the normal inverse Gaussian Lévy process takes the form

$$
L_{t}=\xi t+\int_{0}^{t} \int_{\mathbb{R} \backslash\{0\}} z \tilde{N}(d t, d z), \quad \xi=\mu+\frac{\delta \beta}{\sqrt{a^{2}-\beta^{2}}} .
$$

In empirical studies one usually centres the data and let $\mu=0$. In this case the Lévyr measure takes the form

For $z \geq 1$, we have

$$
\nu(d z)=\frac{\alpha \delta}{\pi|z|} e^{\beta z} K_{1}(\alpha|z|) d z .
$$

$$
\left(e^{z}-1\right) \exp \left(-\frac{1}{2} \alpha z\left(x+x^{-1}\right)\right) \leq \exp \left(-\frac{1}{2}(\alpha-1) z\left(x+x^{-1}\right)\right),
$$

since $x+x^{-1} \geq 2$ for positive $x$. By adjusting the $\alpha$ - parameter to $\alpha-1$, we have that $\left(e^{z}-1\right) \nu(d z)$ for $z \geq 1$ is dominated by another Lévy measure coming from a normal inverse Gaussian Lévy process. On the other hand, when $z \leq-1$, we know that $\left|e^{z}-1\right| \leq 1$. Since all Lévy measures integrate 1 for $|z| \geq 1$, we have that (2.4) holds whenever $\alpha>1$. In conclusion, when $\alpha>1$, the normal inverse Gaussian Lévy process satisfies (2.4).

\footnotetext{
${ }^{1}$ The parameters $\mu, \beta$, and $\delta$ are unrelated to the those used in the control problem. The notation of the parameters used lreve are simply chosen to be consistent with the notation in $[6,30]$.
} 
We recall from empirical studies by Rydberg [30] that the estimated $\alpha$ for two German and two Danish stocks were far greater than 1. For instance, the estimated parameter's of Deutsche Bank for day-to-day ticks in the period October 1st, 1989 to December 29th, 1995 (1562 data points) were $(\alpha, \beta, \delta)=(75.49,-4.089,0.012)$. We conclude that a stock price model $S_{t}$ for Deutsche Bank, where the logreturns are modelled by a normal inverse Gaussian distribution with the parameters above, will fit the framework presented in this paper.

\section{Properties of the value function}

In this section we prove that the value function $V$ defined in (2.8) possesses certain growth, monotonicity, and regularity properties. The proofs of these results are inspired by the proofs of the corresponding results in [1].

Lemma 3.1. The value function $V$ is well defined in $\overline{\mathcal{D}}$ and satisfies $0 \leq V(x, y) \leq K(1+x+y)^{\gamma}$ in $\overline{\mathcal{D}}$. Furthermore, $V(x, y)$ is nondecreasing and concave in $\overline{\mathcal{D}}$.

Proof. The arguments used to prove that $V$ is nondecreasing and concave on its convex domain are classical (see, e.g., [1]) and thus omitted. We concentrate here on the growth condition.

First, observe that for every $x, y \in \overline{\mathcal{D}}, \mathcal{A}_{x, y}$ is nonempty. This is so because for every $\pi_{t}, X_{t}^{\pi, 0}$ is obviously nonnegative. Moreover, since the associated gain

$$
\int_{0}^{\infty} e^{-\delta t} U\left(y e^{-\beta t}\right) d t
$$

is nomnegative, $V$ is also nonnegative. The upper bound is established in the following manner, Let $y>0$ and $\pi, C \in \mathcal{A}_{x, y}$. For $n>0$, consider the stopping time $\tau_{n}=\inf \left\{t \geq 0: X_{t}^{\pi, C}>n\right\}$. The process

$$
Z_{t}=X_{t}+\frac{Y_{t}}{\beta}
$$

is bounded away from zero since $Y_{t} \geq y e^{-\beta t}$. Moreover, $Z_{t}$ is a solution of

$$
d Z_{t}=\left[\left(r+(\hat{\mu}-r) \pi_{t}\right) X_{t}-Y_{t}\right] d t+\pi_{t-} X_{t-} \int_{\mathbb{R} \backslash\{0\}}\left(e^{z}-1\right) \tilde{N}(d t, d z)
$$

with initial value $z=x+y / \beta$. Applying Itô's formula, the nomnegativity of $X_{t}, Y_{t}$, and the observation that $\frac{X_{t}}{Z_{t}}, \pi_{t} \frac{X_{t}}{Z_{t}} \in[0,1]$, we obtain

$$
\begin{aligned}
\mathrm{E}\left[Z_{t \wedge \tau_{n}}^{\gamma}\right] & =z^{\gamma}+\gamma \mathrm{E}\left[\int_{0}^{t \wedge \tau_{n}} Z_{s}^{\gamma-1}\left(\left(r+(\hat{\mu}-r) \pi_{s}\right) X_{s}-Y_{s}\right) d s\right] \\
& +\mathrm{E}\left[\int_{0}^{t \wedge \tau_{n}}\left(\int_{\mathbb{R} \backslash\{0\}}\left(\left(Z_{s}+\pi_{s} X_{s}\left(e^{z}-1\right)\right)^{\gamma}-Z_{s}^{\gamma}-\gamma \pi_{s} Z_{s}^{\gamma-1} X_{s}\left(e^{z}-1\right)\right) \nu(d z)\right) d s\right] \\
& =z^{\gamma}+\gamma \mathrm{E}\left[\int_{0}^{t \wedge \tau_{n}} Z_{s}^{\gamma}\left(\left(r+(\hat{\mu}-r) \pi_{s}\right) \frac{X_{s}}{Z_{s}}-\frac{Y_{s}}{Z_{s}}\right) d s\right] \\
& +\mathrm{E}\left[\int_{0}^{t \wedge \tau_{n}} Z_{s}^{\gamma}\left(\int_{\mathbb{R} \backslash\{0\}}\left(\left(1+\left(\pi_{s} \frac{X_{s}}{Z_{s}}\right)\left(e^{z}-1\right)\right)^{\gamma}-1-\gamma\left(\pi_{s} \frac{X_{s}}{Z_{s}}\right)\left(e^{z}-1\right)\right) \nu(d z)\right) d s\right] \\
& \leq z^{\gamma}+\mathrm{E}\left[\int _ { 0 } ^ { t \wedge \tau _ { n } } Z _ { s } ^ { \gamma } \left(\gamma\left(r+(\hat{\mu}-r)\left(\pi_{s} \frac{X_{s}}{Z_{s}}\right)\right)\right.\right. \\
& \left.\left.+\int_{\mathbb{R} \backslash\{0\}}\left(\left(1+\left(\pi_{s} \frac{X_{s}}{Z_{s}}\right)\left(e^{z}-1\right)\right)^{\gamma}-1-\gamma\left(\pi_{s} \frac{X_{s}}{Z_{s}}\right)\left(e^{z}-1\right)\right) \nu(d z)\right) d s\right] \\
& \leq z^{\gamma}+\mathrm{E}\left[\int_{0}^{t \wedge \tau_{n}} Z_{s}^{\gamma} d s\right] k(\gamma),
\end{aligned}
$$

where $k(\gamma)$ is defined in (2.9). Gronwall's lemma now yields $\mathrm{E}\left[Z_{t \wedge \tau_{n}}^{\gamma}\right] \leq z^{\gamma} e^{k(\gamma) t}$. Letting $n \rightarrow \infty$, we have by Fatou's lemma that

$$
\mathrm{E}\left[Y_{t}^{\gamma}\right] \leq K(x+y)^{\gamma} e^{k(\gamma) t} .
$$


Note that this bound also holds when $y=0$ by continuity. The growth condition on the utility function $U$ then implies that (recall $\delta>k(\gamma)$ )

$$
\mathrm{E}\left[\int_{0}^{\infty} e^{-\delta t} U\left(Y_{t}\right) d t\right] \leq K \int_{0}^{\infty} e^{-\delta t}\left[1+(x+y)^{\gamma} e^{k(\gamma) t}\right] d t \leq K(1+x+y)^{\gamma}
$$

Maximizing over $\mathcal{A}_{x, y}$ yields the desired upper bound.

Theorem 3.1. The value function $V$ is uniformly continuous in $\overline{\mathcal{D}}$. If for some $\alpha \in(0,1]$, we have $\delta>k(\alpha)$ and $U \in C^{0, \alpha}([0, \infty))$, then $V \in C^{0, \alpha}(\overline{\mathcal{D}})$. Furthermore, if $\delta>k(1+\alpha)$ and $U \in C^{1, \alpha}([0, \infty))$, then $V \in C^{1, \alpha}(\overline{\mathcal{D}})$.

Proof. We first show how to compare admissible trajectories starting from different points. For $(x, y),\left(x^{\prime}, y^{\prime}\right) \in \overline{\mathcal{D}}$, let $\pi, C \in \mathcal{A}_{: x, y}$ and define the stopping time

$$
\tau=\inf \left\{t \geq 0: X_{t}^{\prime \pi, C}<0\right\}
$$

We use the short-hand notation $X_{t}^{\prime}$ for the process starting at $x^{\prime}$. When $x^{\prime} \geq x$ we observe that $\tau=\infty$. Set

$$
\begin{aligned}
& C_{t}^{\prime}=C_{t} \mathbf{1}_{t<\tau}+\left(\Delta X_{\tau}^{\prime \pi, C}+X_{\tau-}^{\prime \pi, C}+C_{\tau}\right) \mathbf{1}_{t \geq \tau} \\
& \Gamma_{t}=C_{t}-C_{t}^{\prime}=\left(C_{t}-\Delta X_{\tau}^{\prime \pi, C}-X_{\tau-}^{\prime \pi, C}-C_{\tau}\right) \mathbf{1}_{t \geq \tau}
\end{aligned}
$$

We see that $C_{t}^{\prime}=C_{t}$ and $\Gamma_{t}=0$ when $x^{\prime} \geq x$. Since

$$
\Delta X_{\tau}^{\prime \pi, C}=-\Delta C_{\tau}^{\prime}+\pi_{\tau-} X_{\tau-}^{\prime \pi, C}\left(e^{\Delta L_{\tau}}-1\right)
$$

and $X_{\tau-}^{\prime \pi, C} \geq 0$, we call show that

$$
\Delta X_{\tau}^{\prime \pi, C}+X_{\tau-}^{\prime \pi, C}+C_{\tau} \geq C_{\tau-}^{\prime}+\left(1-\pi_{\tau-}\right) X_{\tau-}^{\prime \pi, C} \geq C_{\tau-} .
$$

We immediately see from this and (3.2) that $C_{t}^{\prime}$ is nondecreasing. Similarly we have that $\Gamma$ is nondecreasing. We now calculate

$$
\begin{aligned}
X_{t}^{\prime \pi, C} \mathbf{1}_{t<\tau}= & X_{t \wedge \tau}^{\prime \pi, C}-\left(\Delta X_{\tau}^{\prime \pi, C}+X_{\tau-}^{\prime \pi, C}\right) \mathbf{1}_{t \geq \tau} \\
= & x^{\prime}-C_{t}^{\prime}+\int_{0}^{t \wedge \tau}\left(r+(\hat{\mu}-r) \pi_{s}\right) X_{s}^{\prime \pi, C} d s+\int_{0}^{t \wedge \tau} \pi_{s-} X_{s-}^{\prime \pi, C} \int_{\mathbb{R} \backslash\{0\}}\left(e^{z}-1\right) \tilde{N}(d s, d z) \\
= & x^{\prime}-C_{t}^{\prime}+\int_{0}^{t}\left(r+(\hat{\mu}-r) \pi_{s}\right) X_{s}^{\prime \pi, C} \mathbf{1}_{s<\tau} d s \\
& \quad+\int_{0}^{t} \pi_{s-}\left(X^{\prime \pi, C} \mathbf{1}_{<<\tau}\right)_{s-} \int_{\mathbb{R} \backslash\{0\}}\left(e^{z}-1\right) \tilde{N}(d s, d z) .
\end{aligned}
$$

By uniqueness we have $X_{t}^{\prime \pi, C^{\prime}}=X_{t}^{\prime \pi, C} 1_{t<\tau}$. This implies that $X_{t}^{\prime \pi, C^{\prime}} \geq 0$ and, when $x>x^{\prime}$,

$$
\left(X-X^{\prime}\right)_{t}^{\pi, \Gamma}=X_{t}^{\pi, C}-X_{t}^{\prime \pi, C^{\prime}}=X_{t}^{\pi, C}-X_{t}^{\prime \pi, C} \mathbf{1}_{t<\tau} \geq 0 .
$$

This in particular leads to the conclusion that $\pi, C^{\prime} \in \mathcal{A}_{x^{\prime}, y^{\prime}}$ and $\pi, \Gamma \in \mathcal{A}_{\left|x-x^{\prime}\right|,\left|y-y^{\prime}\right| \text {. Note that }}$ this is trivial when $x^{\prime} \geq x$. From the explicit form of $Y_{t}$, we get

$$
\left|Y_{t}^{\pi, C}-Y_{t}^{\prime \pi, C^{\prime}}\right| \leq\left|Y-Y^{\prime}\right|_{t}^{\pi, \Gamma}
$$

and thus

$$
\begin{aligned}
\mathrm{E}\left[\int_{0}^{\infty} e^{-\delta t} U\left(Y_{t}^{\pi, C}\right) d t\right] & \leq \mathrm{E}\left[\int_{0}^{\infty} e^{-\delta t} U\left(Y_{t}^{\prime \pi, C^{\prime}}\right) d t\right]+\mathrm{E}\left[\int_{0}^{\infty} e^{-\delta t} \omega_{U}\left(\left|Y-Y^{\prime}\right|_{t}^{\pi, \Gamma}\right) d t\right] \\
& \leq V\left(x^{\prime}, y^{\prime}\right)+\omega_{V}\left(\left|x-x^{\prime}\right|,\left|y-y^{\prime}\right|\right)
\end{aligned}
$$

where $\omega_{U}$ denotes a modulus of continuity for $U$. We have used the notation $\omega_{V}$ for the value function when we replace $U$ by $\omega_{U}$. Maximizing over $\mathcal{A}_{x, y}$ and exchanging $x, y$ and $x^{\prime}, y^{\prime}$, we obtain

$$
\left|V(x, y)-V\left(x^{\prime}, y^{\prime}\right)\right| \leq \omega_{V}\left(\left|x-x^{\prime}\right|,\left|y-y^{\prime}\right|\right)
$$


In the case $U \in C^{0, \alpha}([0, \infty))$, we choose $\omega_{U}(z)=K z^{\alpha}$ and, since $\delta>k(\alpha)$, we conclude from (3.1) that $\omega_{V} \leq K(x+y)^{\alpha}$ in $\overline{\mathcal{D}}$. Hence $V \in C^{0, \alpha}(\overline{\mathcal{D}})$. In general, we choose

$$
\omega_{U}(z)=\inf _{\varepsilon>0}\left(\varepsilon+K_{\varepsilon} z^{\gamma}\right)
$$

and obtain $\omega_{V}(z) \leq \inf _{\varepsilon>0}\left(\varepsilon+K_{\varepsilon} z^{\gamma}\right)$ since $\delta>k(\gamma)$. This implies that $V$ is uniformly continuous.

We now prove the $C^{1, \alpha}$ regularity. Let $\left(x^{\prime}, y^{\prime}\right),\left(x^{\prime \prime}, y^{\prime \prime}\right) \in \overline{\mathcal{D}}$ and define

$$
(x, y)=\left(\lambda x^{\prime}+(1-\lambda) x^{\prime \prime}, \lambda y^{\prime}+(1-\lambda) y^{\prime \prime}\right) \text { for } \lambda \in(0,1) .
$$

Without loss of generality, we assume that $x^{\prime} \leq x \leq x^{\prime \prime}$. Let now $\pi, C \in \mathcal{A}_{x, y}$. Define

$$
C_{t}^{\prime \prime}=C_{t}+\frac{\lambda}{1-\lambda} \Gamma_{t}, \quad \Gamma_{t}^{\prime}=\frac{1}{1-\lambda} \Gamma_{t}
$$

where $C_{t}^{\prime}$ and $\Gamma_{t}$ are defined in (3.2). By linearity we see that for $\pi, \Gamma \in \mathcal{A}_{x-x^{\prime},\left|y-y^{\prime}\right|}$,

$$
\left(X^{\prime \prime}-X^{\prime}\right)_{t}^{\pi, \Gamma^{\prime}}=\frac{1}{1-\lambda}\left(X-X^{\prime}\right)_{t}^{\pi, \Gamma} \geq 0 .
$$

We also find

$$
X_{t}^{\prime \prime \pi, C^{\prime \prime}}=X_{t}^{\pi, C}+\lambda\left(X^{\prime \prime}-X\right)_{t}^{\pi, \Gamma^{\prime}} \geq 0
$$

Therefore $\pi, C^{\prime \prime} \in \mathcal{A}_{x^{\prime \prime}, y^{\prime \prime}}$ and $\pi, \Gamma^{\prime} \in \mathcal{A}_{\left|x^{\prime}-x^{\prime \prime}\right|,\left|y^{\prime}-y^{\prime \prime}\right|}$.

Since $U \in C^{1, \alpha}([0, \infty))$, one can show that

$$
U\left(\lambda z^{\prime}+(1-\lambda) z^{\prime \prime}\right)-\lambda U\left(z^{\prime}\right)-(1-\lambda) U\left(z^{\prime \prime}\right) \leq \lambda(1-\lambda) K\left|z^{\prime}-z^{\prime \prime}\right|^{1+\alpha}
$$

A straightforward calculation gives $Y_{t}^{\pi, C}=\lambda Y_{t}^{\prime} \pi, C^{\prime}+(1-\lambda) Y_{t}^{\prime \prime \pi, C^{\prime \prime}}$. Hence,

$$
\begin{aligned}
\mathrm{E}\left[\int_{0}^{\infty} e^{-\delta t} U\left(Y_{t}^{\pi, C}\right) d t\right]-\lambda V\left(x^{\prime}, y^{\prime}\right)-(1-\lambda) V\left(x^{\prime \prime}, y^{\prime \prime}\right) \leq \mathrm{E}\left[\int_{0}^{\infty} e^{-\delta t} U\left(Y_{t}^{\pi, C}\right) d t\right] \\
\quad-\lambda \mathrm{E}\left[\int_{0}^{\infty} e^{-\delta t} U\left(Y_{t}^{\prime \prime} \pi, C^{\prime \prime}\right) d t\right]-(1-\lambda) \mathrm{E}\left[\int_{0}^{\infty} e^{-\delta t} U\left(Y_{t}^{\prime \prime \pi, C^{\prime \prime}}\right) d t\right] \\
\quad \leq \lambda(1-\lambda) K \mathrm{E}\left[\int_{0}^{\infty} e^{-\delta t}\left|Y_{t}^{\prime}-Y_{t}^{\prime \prime}\right|^{1+\alpha} d t\right] \\
\quad \leq \lambda(1-\lambda) K\left(\left|x^{\prime}-x^{\prime \prime}\right|+\left|y^{\prime}-y^{\prime \prime}\right|\right)^{1+\alpha} .
\end{aligned}
$$

The last inequality follows from (3.1) because $\delta>k(1+\alpha)$ by assumption and $\pi, \Gamma^{\prime} \in \mathcal{A}_{\mid x^{\prime}-x^{\prime \prime}}|,| y^{\prime}-y^{\prime \prime} \mid$. By maximizing over $\mathcal{A}_{x, y}$ and using the concavity of $V$ we get

$$
0 \leq V(x, y)-\lambda V\left(x^{\prime}, y^{\prime}\right)-(1-\lambda) V\left(x^{\prime \prime}, y^{\prime \prime}\right) \leq \lambda(1-\lambda) K\left(\left|x^{\prime}-x^{\prime \prime}\right|+\left|y^{\prime}-y^{\prime \prime}\right|\right)^{1+\alpha} .
$$

This inequality holds for all $\left(x^{\prime}, y^{\prime}\right),\left(x^{\prime \prime}, y^{\prime \prime}\right) \in \overline{\mathcal{D}}$ and $\lambda \in(0,1)$. Hence $V \in C^{1, \alpha}(\overline{\mathcal{D}})$.

\section{Viscosity SOLUTIONS}

In this section we characterise the value function (2.8) as the unique constrained viscosity solution of the integro-differential variational inequality (2.11). To simplify the presentation, we will on several occasions employ the following notations: $X=\left(x_{1}, x_{2}\right) \in \overline{\mathcal{D}}, D_{X}=\left(\partial_{x_{1}}, \partial_{x_{2}}\right)$, $G\left(D_{X} v\right)=\beta v_{x_{2}}-v_{x_{1}}$, and

$$
\begin{aligned}
& \mathcal{B}^{\pi}(X, v)=\int_{\mathbb{R} \backslash\{0\}}\left(v\left(x_{1}+x_{1} \pi\left(e^{z}-1\right), x_{2}\right)-v\left(x_{1}, x_{2}\right)-\pi x_{1} v_{x_{1}}(X)\left(e^{z}-1\right)\right) \nu(d z), \\
& F\left(X, v, D_{X} v, \mathcal{B}^{\pi}(X, v)\right)=U\left(x_{2}\right)-\delta v-\beta x_{2} v_{x_{2}}+\max _{\pi \in[0,1]}\left[(v+(\hat{\mu}-v) \pi) x_{1} v_{x_{1}}+\mathcal{B}^{\pi}(X, v)\right] .
\end{aligned}
$$

Then (2.11) takes the following form

$$
\max \left(G\left(D_{X} v\right) ; F\left(X, v, D_{X} v, \mathcal{B}^{\pi}(X, v)\right)\right)=0 \text { in } \mathcal{D} .
$$


Recall that the Lévy measure $\nu(d z)$ is a positive $\sigma$-finite measure on $\mathbb{R} \backslash\{0\}$ with a possible singularity in zero so that (2.2) holds. We thus need to be more specific about the meaning of the integro-differential operator $\mathcal{B}^{\pi}$. To this end, define the set

$$
C_{\ell}(\overline{\mathcal{D}})=\left\{\phi \in C(\overline{\mathcal{D}}): \sup _{\overline{\mathcal{D}}} \frac{|\phi(X)|}{\left(1+x_{1}+x_{2}\right)^{\ell}}<\infty\right\}, \quad \ell \geq 0 .
$$

For any $\kappa \in(0,1), X \in \overline{\mathcal{D}}, \phi \in C_{1}(\overline{\mathcal{D}}), P=\left(p_{1}, p_{2}\right) \in \mathbb{R}^{2}$, we define

$$
\mathcal{B}^{\pi, \kappa}(X, \phi, P)=\int_{|z|>\kappa}\left(\phi\left(x_{1}+x_{1} \pi\left(e^{z}-1\right), x_{2}\right)-\phi(X)-\pi x_{1} p_{1}\left(e^{z}-1\right)\right) \nu(d z) .
$$

The integrand of $\mathcal{B}^{\pi, \kappa}(X, \phi, P)$ is bounded by

$$
\text { Const }(X, P, \kappa) \cdot\left(1+\left|e^{z}-1\right|\right)
$$

and, thanks to (2.4), the integral is convergent and bounded uniformly in $\pi$ for every positive $\kappa$. For $\kappa \in(0,1), X \in \overline{\mathcal{D}}, \phi \in C^{2}(\overline{\mathcal{D}})$, we define

$$
\mathcal{B}_{\kappa}^{\pi}(X, \phi)=\int_{|z| \leq k}\left(\phi\left(x_{1}+x_{1} \pi\left(e^{z}-1\right), x_{2}\right)-\phi(X)-\pi x_{1} \phi_{x_{1}}(X)\left(e^{z}-1\right)\right) \nu(d z) .
$$

Note that $\phi\left(x_{1}+x_{1} \pi\left(e^{z}-1\right), x_{2}\right)=\phi(X)+\phi_{x_{1}}(X)\left(x_{1} \pi\left(e^{z}-1\right)\right)+\frac{1}{2} \phi_{x_{1} x_{1}}\left(a, x_{2}\right)\left(x_{1} \pi\left(e^{z}-1\right)\right)^{2}$, where $a$ is some point on the line between $X$ and $\left(x_{1}+x_{1} \pi\left(e^{z}-1\right), x_{2}\right)$. Hence the integrand of $\mathcal{B}_{\kappa}^{\pi}(X, \phi)$ is bounded by

$$
\text { Const }(X, \kappa) \cdot\left|e^{z}-1\right|^{2}
$$

and the integral is convergent and bounded uniformly in $\pi$ since every Lévy measure integrates $z^{2}$ in a neighbourhood of zero, see (2.2). Furthermore,

$$
\lim _{\kappa \rightarrow 0+} B_{\kappa}^{\pi}(X, \phi)=0 \text {. }
$$

We now define for all $\phi \in C^{2}(\overline{\mathcal{D}}) \cap C_{1}(\overline{\mathcal{D}})$ the integro-differential operator $\mathcal{B}^{\pi}(X, \phi)$ by

$$
\mathcal{B}^{\pi}(X, \phi):=\mathcal{B}^{\pi, k}\left(X, \phi, D_{X} \phi\right)+\mathcal{B}_{k}^{\pi}(X, \phi) \text {. }
$$

Consequently, the Hamilton-Jacobi-Bellman equation (4.1) is well defined for all $v \in C^{2}(\overline{\mathcal{D}}) \cap$ $C_{1}(\overline{\mathcal{D}})$. However, in many applications the value function defined in $(2.8)$ is not $C^{2}$ or even $C^{1}$ (see Section 3) and the equation (4.1) should be interpreted in a weaker sense. As discussed in Section 1, we here suitably adopt the notion of constrained viscosity solutions. Constrained viscosity solutions are functions that are supersolutions of (2.11) in $\mathcal{D}$ and subsolutions of (2.11) in $\overline{\mathcal{D}}$. The latter requirement plays the role of a boundary condition, see $[33,34,13]$.

The precise definition goes as follows:

Definition 4:1. (i) Let $\mathcal{O} \subset \overline{\mathcal{D}}$. Any $v \in C(\overline{\mathcal{D}})$ is a viscosity subsolution (supersolution) of (4.1) in $\mathcal{O}$ if and only if we have, for every $X \in \mathcal{O}$ and $\phi \in C^{2}(\overline{\mathcal{D}}) \cap C_{1}(\overline{\mathcal{D}})$ such that $X$ is a global maximum (minimum) relative to $\mathcal{O}$ of $v-\phi$,

$$
\max \left(G\left(D_{X} \phi\right) ; F\left(X, v, D_{X} \phi, \mathcal{B}^{\pi}(X, \phi)\right)\right) \geq 0(\leq 0) .
$$

(ii) Any $v \in C(\overline{\mathcal{D}})$ is a constrained viscosity solution of (4.1) if and only if $v$ is a viscosity supersolution of (4.1) in $\mathcal{D}$ and $v$ is a viscosity subsolution of (4.1) in $\overline{\mathcal{D}}$.

Hereafter we use the terms subsolution and supersolution instead of viscosity subsolution and viscosity supersolution. For $\kappa>0, \phi \in C^{2}(\overline{\mathcal{D}}), v \in C_{1}(\overline{\mathcal{D}})$ let us introduce the function

$$
\begin{aligned}
& F\left(X, v, D_{X} \phi, \mathcal{B}^{\pi, \kappa}\left(X, v, D_{X} \phi\right), \mathcal{B}_{k}^{\pi}(X, \phi)\right) \\
& \quad=U\left(x_{2}\right)-\delta v-\beta x_{2} \phi_{x_{2}}+\max _{\pi \in[0,1]}\left[(r+(\hat{\mu}-r) \pi) x_{1} \phi_{x_{1}}+\mathcal{B}^{\pi, k}\left(X, v, D_{X} \phi\right)+\mathcal{B}_{k}^{\pi}(X, \phi)\right] .
\end{aligned}
$$

Note that $\mathcal{B}^{\pi, i}\left(X, v, D_{X} \phi\right)$ and $\mathcal{B}_{f_{i}}^{\pi}(X, \phi)$ are well defined and bounded independently of $\pi$.

We now have an equivalent formulation of viscosity solutions in $C_{1}(\overline{\mathcal{D}})$. 
Lemma 4.1. Let $v \in C_{1}(\overline{\mathcal{D}})$ and $\mathcal{O} \subset \overline{\mathcal{D}}$. Then $v$ is a subsolution (supersolution) of (4.1) in $\mathcal{O}$ if and only if we have, for every $\phi \in C^{2}(\overline{\mathcal{D}})$ and $\kappa>0$,

$$
\max \left(G\left(D_{X} \phi\right) ; F\left(X, v, D_{X} \phi, \mathcal{B}^{\pi, \kappa}\left(X, v, D_{X} \phi\right), \mathcal{B}_{k}^{\pi}(X, \phi)\right)\right) \geq 0(\leq 0)
$$

whenever $X \in \mathcal{O}$ is a global maximum (minimum) relative to $\mathcal{O}$ of $v-\phi$.

Proof. We prove the statement only for the subsolutions, the supersolution case can be proved similarly. Suppose $v \in C_{1}(\overline{\mathcal{D}})$ satisfies

$$
F\left(X, v, D_{X} \phi, \mathcal{B}^{\pi, \kappa}\left(X, v, D_{X} \phi\right), \mathcal{B}_{k}^{\pi}(X, \phi)\right) \geq 0
$$

where $X \in \mathcal{O}$ is a global maximum relative to $\mathcal{O}$ of $v-\phi, \phi \in C^{2}(\overline{\mathcal{D}}) \cap C_{1}(\overline{\mathcal{D}})$. Then, since $X \in \mathcal{O}$ is a global maximum, $v(Y)-v(X) \leq \phi(Y)-\phi(X)$ for all $Y \in \mathcal{O}$. Consequently, since $\mathcal{B}^{\pi, \kappa}\left(X, \phi, D_{X} \phi\right) \geq \mathcal{B}^{\pi, k}\left(X, v, D_{X} \phi\right)$, we can use (4.3) and (4.6) to conclude that

$$
F\left(X, v, D_{X} \phi, \mathcal{B}^{\pi}(X ; \phi)\right)=F\left(x, v, D_{X} \phi, \mathcal{B}^{\pi, r}\left(X, \phi, D_{X} \phi\right), \mathcal{B}_{*}^{\pi}(X, \phi)\right) \geq 0
$$

This implies that $v$ is a subsolution of (4.1) in $\mathcal{O}$ if (4.5) holds.

Conversely, let $v \in C_{1}(\overline{\mathcal{D}})$ be a subsolution of (4.1) in $\mathcal{O}$ and assume that $X \in \mathcal{O}$ is a global maximum relative to $\mathcal{O}$ of $v-\phi, \phi \in C^{2}(\overline{\mathcal{D}})$. Let $\chi_{n}$ be a smooth function satisfying $0 \leq \chi_{n} \leq 1$, $\chi_{n}(Y)=1$ for $Y \in \mathcal{N}\left(X, x_{1}\left(e^{\kappa}-1-\frac{1}{n}\right)\right) \cap \mathcal{O}$, and $\chi_{n}(Y)=0$ for $Y \in \mathcal{O} \backslash\left(\mathcal{N}\left(X, x_{1}\left(e^{\kappa}-1\right)\right) \cap \mathcal{O}\right)$. Here $\mathcal{N}(X, R)$ denotes the open ball centred in $X$ with radius $R$. Then define the test function

$$
\psi_{n}(Y)=\chi_{n}(Y) \phi(Y)+\left(1-\chi_{n}(Y)\right) v_{n}(Y) \in C^{2}(\overline{\mathcal{D}}) \cap C_{1}(\overline{\mathcal{D}})
$$

where $v_{n} \in C^{\infty}(\overline{\mathcal{D}})$ is such that $v_{n} \uparrow v$ a.e. in $\mathcal{O} \backslash\left(\mathcal{N}\left(X, x_{1}\left(e^{\kappa}-1\right)\right) \cap \mathcal{O}\right)$ as $n \rightarrow \infty$. Observe that $\psi_{n}=\phi$ in $\mathcal{N}\left(X, x_{1}\left(e^{k}-1-\frac{1}{n}\right)\right) \cap \mathcal{O}, \psi_{n} \rightarrow \phi$ in $\mathcal{N}\left(X, x_{1}\left(e^{\kappa}-1\right)\right) \cap \mathcal{O}, \psi_{n}=v_{n}$ in $\mathcal{O} \backslash\left(\mathcal{N}\left(X, x_{1}\left(e^{r}-1\right)\right) \cap \mathcal{O}\right)$, and $X$ is a global maximum relative to $\mathcal{O}$ of $v-\psi_{n}$. Therefore,

$$
\begin{aligned}
& F\left(X, v, D_{X} \psi_{n}, \mathcal{B}^{\pi}\left(X, \psi_{n}\right)\right)=F\left(X, v, D_{X} \phi, \mathcal{B}^{\pi, \kappa}\left(X, \psi_{n}, D_{X} \psi_{n}\right), \mathcal{B}_{\kappa}^{\pi}\left(X, \psi_{n}\right)\right) \\
& \quad \rightarrow F\left(X, v, D_{X} \phi, \mathcal{B}^{\pi, \kappa}\left(X, v, D_{X} \phi\right), \mathcal{B}_{\kappa}^{\pi}(X, \phi)\right),
\end{aligned}
$$

where we have used Lebesgue's dominated convergence theorem to conclude that

$$
\mathcal{B}^{\pi, k}\left(X, \psi_{n}, D_{X} \psi_{n}\right)=\mathcal{B}^{\pi, \kappa}\left(X, v_{n}, D_{X} \phi\right) \rightarrow \mathcal{B}^{\pi, k}\left(X, v, D_{X} \phi\right), \quad \mathcal{B}_{k}^{\pi}\left(X, \psi_{n}\right) \rightarrow \mathcal{B}_{k}^{\pi}(X, \phi) .
$$

This implies that (4.5) holds if $v \in C_{1}(\overline{\mathcal{D}})$ is a subsolution of (4.1) in $\mathcal{O}$.

It is convenient to use Definition 4.1 when proving existence of a constrained viscosity solution, whereas the formulation based on Lemma 4.1 is more convenient when proving uniqueness. We also note that Lemma 4.1 is an adaption of a similar lemma in Soner [33], see also Sayah [31].

The following easy result will be useful when proving Theorem 4.1 below.

Lemma 4.2. If $\left(x^{\prime}, y^{\prime}\right) \in \overline{\mathcal{D}}$ and $(x, y) \in \overline{\mathcal{D}}$ satisfy $x^{\prime}=x-c$ and $y^{\prime}=y+\beta c$ for some $c>0$, then $V(x, y) \geq V\left(x^{\prime}, y^{\prime}\right)$.

We next characterize $V$ as a viscosity solution of the Hamilton-Jacobi-Bellman equation (2.11).

Theorem 4.1. The value function $V(x, y)$ is a constrained viscosity solution of (2.11).

Proof. We first prove that $\mathrm{V}$ is a supersolution in $\mathcal{D}$. Let $\phi \in C^{2}(\overline{\mathcal{D}}) \cap C_{1}(\overline{\mathcal{D}})$ and $(x, y) \in \mathcal{D}$ be a global minimizer of $V-\phi$. Without any loss of generality we may assume that $(V-\phi)(x, y)=0$. For every $c \in(0, x]$, we choose $C_{0}=c$ and $t=0$ in the dynamic programming principle $(2.10)$, which then yields

$$
\phi(x, y)=V(x, y) \geq V(x-c, y+\beta c) \geq \phi(x-c, y+\beta c) .
$$

Dividing by $c$ and sending $c \rightarrow 0$, we conclude

$$
\phi_{x}(x, y)-\beta \phi_{y}(x, y) \geq 0 .
$$


Let $\tau_{\rho}$ be the exit time from the closed ball $\mathcal{N}_{\rho}$ with radius $\rho$ and centre at $(x, y)$. By choosing $\rho$ small enough, $\mathcal{N}_{\rho} \subset \mathcal{D}$. Applying the dynamic programming principle $(2.10)$ with $h \wedge \tau_{\rho}, \pi_{t}=\pi$, $C_{t}=0$, the inequality $V \geq \phi$, and Itô's formula, we obtain

$$
\begin{aligned}
0 & \geq \mathrm{E}\left[\int_{0}^{h \wedge \tau_{\rho}} e^{-\delta t} U\left(Y_{t}\right) d t+e^{-\delta\left(h \wedge \tau_{\rho}\right)} \phi\left(X_{h \wedge \tau_{\rho}}, Y_{h \wedge \tau_{\rho}}\right)\right]-\phi(x, y) \\
& \geq \mathrm{E}\left[\int_{0}^{h \wedge \tau_{\rho}} e^{-\delta t}\left\{U\left(Y_{t}\right)-\delta \phi-\beta Y_{t} \phi_{y}+(r+(\hat{\mu}-r) \pi) X_{t} \phi_{x}+\mathcal{B}^{\pi}\left(\left(X_{t}, Y_{t}\right), \phi\right)\right\} d t\right] \\
& \geq \mathrm{E}\left[\frac{1-e^{-\delta\left(h \wedge \tau_{\rho}\right)}}{\delta}\right] \inf _{(x, y) \in \mathcal{N}_{\rho}}\left[U(y)-\delta \phi-\beta y \phi_{y}+(r+(\hat{\mu}-r) \pi) x \phi_{x}+\mathcal{B}^{\pi}((x, y), \phi)\right] .
\end{aligned}
$$

By the right continuity of the paths, $\tau_{\rho}>0$ a.s. and hence Lebesgue's dominated convergence theorem implies $\lim _{h \rightarrow 0} \mathrm{E}\left[\frac{1-e^{-\delta\left(h \wedge r_{\rho}\right)}}{h}\right]=\delta$. Dividing the above inequality by $h$, sending $h \rightarrow 0$, and then sending $\rho \rightarrow 0$, we obtain

$$
U(y)-\delta V-\beta y \phi_{y}+(r+(\hat{\mu}-r) \pi) x \phi_{x}+\mathcal{B}^{\pi}((x, y), \phi) \leq 0
$$

for every $\pi \in[0,1]$. Hence, from this and (4.7), we have proven that $V$ is a viscosity supersolution.

We now prove that $V$ is a subsolution in $\overline{\mathcal{D}}$. Let $\phi \in C^{2}(\overline{\mathcal{D}}) \cap C_{1}(\overline{\mathcal{D}})$ and $(x, y) \in \overline{\mathcal{D}}$ be a global maximizer of $V-\phi$. Without any loss of generality we may assume $(V-\phi)(x, y)=0$ and that the maximum is strict. Arguing by contradiction, we suppose that the subsolution inequality (4.4) is violated. Then, by continuity, there is a nonempty open ball $\mathcal{N}$ centred at $(x, y)$ and $\varepsilon>0$ such that $\beta \phi_{y}-\phi_{x} \leq 0$ and

$$
U(y)-\delta V-\beta y \phi_{y}+\max _{\pi \in[0,1]}\left[(r+(\hat{\mu}-r) \pi) x \phi_{x}+\mathcal{B}^{\pi}((x, y), \phi)\right] \leq-\varepsilon \delta \text { in } \overline{\mathcal{N} \cap \mathcal{D}}
$$

as well as $V \leq \phi-\varepsilon$ on $\partial \mathcal{N} \cap \overline{\mathcal{D}}$. For $\pi, C \in \mathcal{A}_{x, y}$, let $\tau^{*}$ be the exit time from $\overline{\mathcal{N}} \cap \mathcal{D}$.

Since $C_{t}$ is a singular control with a possible jump at $t=0$, the state process $\left(X_{t}, Y_{t}\right)$ might jump out of $\overline{\mathcal{N} \cap \mathcal{D}}$ at once. If the control $C_{t}$ alone makes the state process jump out of $\overline{\mathcal{N} \cap \mathcal{D}}$, we know the direction of the jump and, from Lemma 4.2, that $V$ is nonincreasing in this clirection. However, when the Lévy process also contributes to the jump out of $\overline{\mathcal{N} \cap \mathcal{D}}, V$ is not necessarily nonincreasing in the direction of the jump. To overcome this problem we introduce $\tau_{L}$, the first time the state process jumps because of the Lévy process, and note that $\tau_{L}>0$ a.s. Choose $\tau=\min \left(\tau_{L}, \tau^{*}\right)$. If necessary, we truncate this stopping time by a constant in order to make it finite. Let now $A:=\left\{\tau_{L}=0\right\}$ and note that this is a set of zero probability.

On the set $\left\{\tau^{*}<\tau_{L}\right\} \cap A^{c}$, where $A^{c}$ is the complement of $A$, we know that the control $C_{t}$ has made the state process jump out of $\overline{\mathcal{N} \cap \mathcal{D}}$. Let $\left(x^{\prime}, y^{\prime}\right)$ be the intersection between $\partial \mathcal{N} \cap \overline{\mathcal{D}}$ and the line between $\left(X_{\tau^{*}-}, Y_{\tau^{*}-}\right)$ and $\left(X_{\tau^{*}}, Y_{\tau^{*}}\right)$. Note that the slope vector of this line is $(-1, \beta)$ and that $\phi$ is nonincreasing along this line in $\overline{\mathcal{N} \cap \mathcal{D}}$. Thanks to Lemma 4.2, we also know that $V$ is nonincreasing along this line in $\overline{\mathcal{D}}$. Hence we have

$$
V\left(X_{\tau^{*}}, Y_{\tau^{*}}\right) \leq V\left(x^{\prime}, y^{\prime}\right) \leq \phi\left(x^{\prime}, y^{\prime}\right)-\varepsilon \leq \phi\left(X_{\tau^{*}-}, Y_{\tau^{*}-}\right)-\varepsilon
$$


Using the inequalities above and Itô's formula for semimartingales, we obtain (with $C_{t}^{c}$ denoting the continuous part of $C_{t}$ )

$$
\begin{aligned}
& \int_{0}^{\tau^{*}} e^{-\delta t} U\left(Y_{t}\right) d t+e^{-\delta \tau^{*}} V\left(X_{\tau^{*}}, Y_{\tau^{*}}\right) \\
& \leq \int_{U}^{\tau^{*}} e^{-\delta t} U\left(Y_{t}\right) d t+e^{-\delta \tau^{*}} \phi\left(X_{\tau^{*}-}, Y_{\tau^{*}-}\right)-\varepsilon e^{-\delta \tau^{*}} \\
& \leq \phi(x, y)-\varepsilon e^{-\delta \tau^{*}} \\
& +\int_{0}^{\tau^{*}} e^{-\delta t}\left\{U\left(Y_{t}\right)-\delta \phi-\beta Y_{t} \phi_{y}+(r+(\hat{\mu}-r) \pi) X_{t} \phi_{x}+\mathcal{B}^{\pi}\left(\left(X_{t}, Y_{t}\right), \phi\right)\right\} d t \\
& +\int_{0}^{\tau^{*}} e^{-\delta t}\left(-\phi_{x}+\beta \phi_{y}\right) d C_{t}^{c} \\
& +\sum_{\left[0, r^{*}\right)} e^{-\delta t}\left(\phi\left(X_{t-}-\Delta C_{t}, Y_{t-}+\beta \Delta C_{t}\right)-\phi\left(X_{t-}, Y_{t-}\right)\right)+\int_{0}^{r^{*}} \int_{I R \backslash\{0\}} \Delta_{L} \phi \tilde{N}(d t, d z) \\
& \leq \phi(x, y)-\varepsilon e^{\tau^{*}}-\varepsilon\left(1-e^{\tau^{*}}\right)+\int_{0}^{\tau^{*}} \int_{I R \backslash\{0\}} \Delta_{L} \phi \tilde{N}(d t, d z) \\
& \leq \phi(x, y)-\varepsilon+\int_{0}^{\tau^{*}} \int_{\mathbb{R} \backslash\{0\}} \Delta_{L} \phi \tilde{N}(d t, d z),
\end{aligned}
$$

where we have used the short-hand notation

$$
\Delta_{L} \phi=\phi\left(X_{t-}+\pi_{t-} X_{t-}\left(e^{z}-1\right), Y_{t-}\right)-\phi\left(X_{t-}, Y_{t-}\right)
$$

On the set $\left\{\tau * \geq \tau_{L}\right\} \cap A^{c}$, we have $\tau=\tau_{L}$ and calculate as follows

$$
\begin{aligned}
& \int_{0}^{\tau_{L}} e^{-\delta t} U\left(Y_{t}\right) d t+e^{-\delta \tau_{L}} V\left(X_{\tau_{L}}, Y_{\tau_{L}}\right) \\
& \leq \int_{0}^{\tau_{L}} e^{-\delta t} U\left(Y_{t}\right) d t+e^{-\delta \tau_{L}} \phi\left(X_{\tau_{L}}, Y_{\tau_{L}}\right) \\
& \leq \phi(x, y)+\int_{0}^{\tau_{L}} e^{-\delta t}\left\{U\left(Y_{t}\right)-\delta \phi-\beta Y_{t} \phi_{y}+(r+(\hat{\mu}-r) \pi) X_{t} \phi_{x}+\mathcal{B}^{\pi}\left(\left(X_{t}, Y_{t}\right), \phi\right)\right\} d t \\
&+\int_{0}^{\tau_{L}} e^{-\delta t}\left(-\phi_{x}+\beta \phi_{y}\right) d C_{t}^{c} \\
&+\sum_{t \in\left[0, \tau_{L}\right]} e^{-\delta t}\left(\phi\left(X_{t-}-\Delta C_{t}, Y_{t-}+\beta \Delta C_{t}\right)-\phi\left(X_{t-}, Y_{t-}\right)\right)+\int_{0}^{\tau_{L}} \int_{\mathbb{R} \backslash\{0\}} \Delta_{L} \phi \tilde{N}(d t, d z) \\
& \leq \phi(x, y)-\varepsilon\left(1-e^{-\delta \tau_{L}}\right)+\int_{0}^{\tau_{L}} \int_{\mathbb{R} \backslash\{0\}} \Delta_{L} \phi \tilde{N}(d t, d z) .
\end{aligned}
$$

To derive the third inequality in (4.9) from the second, we have assumed that

$$
\left(X_{\tau^{*}-}-\Delta C_{\tau^{*}}, Y_{\tau^{*}-}+\beta \Delta C_{\tau^{*}}\right) \in \overline{\mathcal{N} \cap \mathcal{D}}
$$

In view of Lemma 4.2, we can make such an assumption without loss of generality.

Putting the two cases (4.8) and (4.9) together, we get

$$
\begin{aligned}
& \mathrm{E}\left[\int_{0}^{\tau} e^{-\delta t} U\left(Y_{t}\right) d t+e^{-\delta \tau} V\left(X_{\tau}, Y_{\tau}\right)\right] \\
& \quad \leq \mathrm{E}\left[1_{\tau^{*}<\tau_{L}}\left(\int_{0}^{\tau^{*}} e^{-\delta t} U\left(Y_{t}\right) d t+e^{-\delta \tau^{*}} V\left(X_{\tau^{*}}, Y_{\tau^{*}}\right)\right)\right]
\end{aligned}
$$




$$
\begin{aligned}
& \quad+\mathrm{E}\left[\mathbf{1}_{\tau^{*} \geq \tau_{L}}\left(\int_{0}^{\tau_{L}} e^{-\delta t} U\left(Y_{t}\right) d t+e^{-\delta \tau_{L}} V\left(X_{\tau_{L}}, Y_{\tau_{L}}\right)\right)\right] \\
& \leq \mathrm{E}\left[\mathbf{1}_{\tau^{*}<\tau_{L}}\left(\phi(x, y)-\varepsilon+\int_{0}^{\tau^{*}} \int_{\mathbb{R} \backslash\{0\}} \Delta_{L} \phi \tilde{N}(d t, d z)\right)\right] \\
& \quad+\mathrm{E}\left[\mathbf{1}_{\tau^{*} \geq \tau_{L}}\left(\phi(x, y)-\varepsilon\left(1-e^{-\delta \tau_{L}}\right)+\int_{0}^{\tau_{L}} \int_{\mathbb{R} \backslash\{0\}} \Delta_{L} \phi \tilde{N}(d t, d z)\right)\right] \\
& \leq \phi(x, y)-\varepsilon \mathrm{E}\left[1-\mathbf{1}_{\tau^{*} \geq \tau_{L}} e^{-\delta \tau_{L}}\right]+\mathrm{E}\left[\int_{0}^{\tau} \int_{\mathbb{R} \backslash\{0\}} \Delta_{L} \phi \tilde{N}(d t, d z)\right] \\
& \leq \phi(x, y)-\varepsilon \mathrm{E}\left[1-e^{-\delta \tau_{L}}\right] .
\end{aligned}
$$

The proof is now finished after observing that the dynamic programming principle (2.10) gives a contradiction since $(V-\phi)(x, y)=0$.

Definition 4.2. Let $\mathcal{O} \subset \overline{\mathcal{D}}$. Any $v \in C(\overline{\mathcal{D}})$ is a strict supersolution of (4.1) in $\mathcal{O}$ if and only if we have, for every $X \in \mathcal{O}$ and $\phi \in C^{2}(\overline{\mathcal{D}}) \cap C_{1}(\overline{\mathcal{D}})$ such that $X$ is a global minimum relative to $\mathcal{O}$ of $v-\phi$,

$$
\max \left(G\left(D_{X} \phi\right) ; F\left(X, v, D_{X} \phi, \mathcal{B}^{\pi}(X, \phi)\right)\right) \leq-\nu
$$

for some constant $\nu>0$. Repeating the proof of Lemma 4.1, we can equivalently replace the left-hand side of (4.10) by the left-hand side of (4.5) (provided $v \in C_{1}(\overline{\mathcal{D}})$ and $\phi \in C^{2}(\overline{\mathcal{D}})$ ).

We next demonstrate that it is possible to construct strict supersolutions of (4.1) in any bounded subset of $\mathcal{D}$. To simplify the presentation, we employ the notations provided by (4.1).

Lemma 4.3. For $\gamma^{\prime}>0$ such that $\delta>k\left(\gamma^{\prime}\right)$, let $v \in C_{\gamma^{\prime}}(\overline{\mathcal{D}})$ be a supersolution of $(4.1)$ in $\mathcal{D}$. Choose $\bar{\gamma}>\max \left(\gamma, \gamma^{\prime}\right)$ such that $\delta>k(\bar{\gamma})$, and let

$$
w=K+\chi^{\bar{\gamma}}, \quad \chi(X)=\left(1+x_{1}+\frac{x_{2}}{2 \beta}\right) .
$$

Then for $K$ large enough, $w \in C^{\infty}(\mathcal{D}) \cap C_{\bar{\gamma}}(\overline{\mathcal{D}})$ is a strict supersolution of (4.1) in any bounded set $\mathcal{O} \subset \mathcal{D}$. Moreover, for $\theta \in(0,1]$, the function

$$
v^{\theta}=(1-\theta) v+\theta w \in C_{\bar{\gamma}}(\overline{\mathcal{D}})
$$

is a strict supersolution of (4.1) in any bounded set $\mathcal{O} \subset \mathcal{D}$.

Proof. We first claim that

$$
\max \left(G\left(D_{X} w\right) ; F\left(X, w, D_{X} w, \mathcal{B}^{\pi}(X, w)\right)\right) \leq-f
$$

for some $f \in C(\overline{\mathcal{D}})$ that is strictly positive in any bounded subset of $\mathcal{D}$. Notice that (4.11) implies the first part of Lemma 4.3 .

To prove (4.11), observe first that

$$
G\left(D_{X} w\right)=\beta w_{x_{2}}-w_{x_{1}}=-\frac{\bar{\gamma}}{2} \chi^{\bar{\gamma}-1}
$$

Next, exploiting that $\frac{x_{1}}{\chi}, \pi \frac{x_{1}}{x} \in[0,1]$, we have

$$
\begin{aligned}
F(X, w, & \left.D_{X} w, B(X, w)\right)=U\left(x_{2}\right)-\delta\left(K+\chi^{\bar{\gamma}}\right)-\frac{1}{2} x_{2} \bar{\gamma} \chi^{\bar{\gamma}-1}+\max _{\pi \in[0,1]}\left[\bar{\gamma}(r+(\hat{\mu}-r) \pi) x_{1} \chi^{\bar{\gamma}-1}\right. \\
& \left.+\int_{\mathbb{R} \backslash\{0\}}\left(\left(\chi+\pi x_{1}\left(e^{z}-1\right)\right)^{\bar{\gamma}}-\chi^{\bar{\gamma}}-\bar{\gamma} \pi x_{1} \chi^{\bar{\gamma}-1}\left(e^{z}-1\right)\right) \nu(d z)\right] \\
= & U\left(x_{2}\right)-\delta K-\frac{1}{2} x_{2} \bar{\gamma} \chi^{\bar{\gamma}-1}+\left(-\delta+\max _{\pi \in[0,1]}\left[\bar{\gamma}(r+(\hat{\mu}-r) \pi) \frac{x_{1}}{\chi}\right.\right. \\
& \left.\left.+\int_{\mathbb{R} \backslash\{0\}}\left(\left(1+\pi \frac{x_{1}}{\chi}\left(e^{z}-1\right)\right)^{\bar{\gamma}}-1-\bar{\gamma} \pi \frac{x_{1}}{\chi}\left(e^{z}-1\right)\right) \nu(d z)\right]\right) \chi^{\bar{\gamma}} \\
\leq & U\left(x_{2}\right)-\delta K+\left(-\delta+\max _{\pi \in[0,1]}[\bar{\gamma}(r+(\hat{\mu}-r) \pi)\right.
\end{aligned}
$$




$$
\begin{aligned}
& \left.\left.+\int_{\mathbb{R} \backslash\{0\}}\left(\left(1+\pi\left(e^{z}-1\right)\right)^{\bar{\gamma}}-1-\bar{\gamma} \pi\left(e^{z}-1\right)\right) \nu(d z)\right]\right) \chi^{\bar{\gamma}} \\
= & U\left(x_{2}\right)-\delta K+(k(\bar{\gamma})-\delta) \chi^{\bar{\gamma}} \leq-1
\end{aligned}
$$

by choosing, e.g.,

$$
\delta K=1+\sup _{\overline{\mathcal{D}}}\left[U\left(x_{2}\right)-(\delta-k(\bar{\gamma})) \chi^{\bar{\gamma}}\right]
$$

Note that $\delta K<\infty$ since $\delta>k(\bar{\gamma})$ and $\bar{\gamma}>\gamma$. Consequently, our claim (4.11) holds provided we set

$$
f=\min \left(1, \frac{\bar{\gamma}}{2} \chi^{\bar{\gamma}-1}\right) \text {. }
$$

Next, we claim that $v^{\theta}$ is a strict supersolution of (4.1) in $\mathcal{D}$. Note that for any $\phi \in C^{2}, X \in \mathcal{D}$ is a global minimum of $v-\phi$ if and only if $X$ is a global minimum of $v^{\theta}-\phi^{\theta}$, where $\phi^{\theta}=(1-\theta) \phi+\theta w$. First, since $v$ is a supersolution of (4.1) in $\mathcal{D}$, we have $G\left(D_{X} \phi\right)=\beta \phi_{x_{2}}-\phi_{x_{1}} \leq 0$ and hence

$$
G\left(D_{X} \phi^{\theta}\right)=(1-\theta)\left(\beta \phi_{x_{2}}-\phi_{x_{1}}\right)+\theta\left(\beta w_{x_{2}}-w_{x_{1}}\right) \leq-\theta \frac{\bar{\gamma}}{2} \chi^{\bar{\gamma}-1} .
$$

Letting $\pi^{*} \in[0,1]$ be a maximizer of $(r+(\hat{\mu}-r) \pi) x_{1} \phi_{x_{1}}^{\theta}+\mathcal{B}^{\pi}\left(X, \phi^{\theta}\right)$, we can calculate as follows

$$
\begin{aligned}
F( & \left.X, v^{\theta}, D_{X} \phi^{\theta}, \mathcal{B}^{\pi}\left(X, v^{\theta}\right)\right)=(1-\theta) U\left(x_{2}\right)-\delta(1-\theta) v-\beta x_{2}(1-\theta) \phi_{x_{2}} \\
& +\left(r+(\hat{\mu}-r) \pi^{*}\right) x_{1}(1-\theta) \phi_{x_{1}}+(1-\theta) \mathcal{B}^{\pi^{*}}(X, \phi) \\
& +\theta U\left(x_{2}\right)-\delta \theta w-\beta x_{2} \theta w_{x_{2}}+\left(r+(\hat{\mu}-r) \pi^{*}\right) x_{1} \theta w_{x_{1}}+\theta \mathcal{B}^{\pi^{*}}(X, w) \\
\leq & (1-\theta) F\left(X, v, D_{X} \phi, \mathcal{B}^{\pi}(X, \phi)\right)+\theta F\left(X, w, D_{X} w, \mathcal{B}^{\pi}(X, w)\right) \leq-\theta f .
\end{aligned}
$$

Summing up, we have just shown that

$$
\max \left(G\left(D_{X} \phi^{\theta}\right) ; F\left(X, v^{\theta}, D_{X} \phi^{\theta}, \mathcal{B}^{\pi}\left(X, \phi^{\theta}\right)\right) \leq-\theta f\right.
$$

This concludes the proof of the lemma.

Following the general viscosity solution technique $[11,9,10]$, we next present a comparison principle for constrained viscosity solutions of our integro-differential variational inequality (2.11). This comparison principle immediately implies that the value function defined in (2.8) is the only solution of (2.11). For orientation, we mention once more that the comparison results in $[34,35,31,32,2,29]$ do not apply in our context. Having said this, we do not hesitate to point out that our comparison principle is nevertheless inspired by these results.

To handle the gradient constraint, we use Lemma 4.3 to produce strict supersolutions that are close to the supersolution being compared. Although in the proof of Lemma 4.3, the right-hand side $f=f(x)$ is continuous and strictly positive on bounded subsets of $\mathcal{D}$, it is not uniformly bounded away from zero as $x \rightarrow \infty$. However, this will not cause problems as we manage to reduce the comparison proof to analysis in a bounded set. To handle the state constraint boundary condition, we adapt an idea of Soner $[33,34]$.

In what follows, we use again the notations provided by (4.1) to simplify the presentation.

Theorem 4.2. Let $\gamma^{\prime}>0$ be such that $\delta>k\left(\gamma^{\prime}\right)$. Assume $\underline{v} \in C_{\gamma^{\prime}}(\overline{\mathcal{D}})$ is a subsolution of (4.1) in $\overline{\mathcal{D}}$ and $\bar{v} \in C_{\gamma^{\prime}}(\overline{\mathcal{D}})$ is a supersolution of (4.1) in $\mathcal{D}$. Then $\underline{v} \leq \bar{v}$ in $\overline{\mathcal{D}}$.

Proof. Choose $\bar{\gamma}>\gamma^{\prime}$ such that $\delta>k(\bar{\gamma})$ and then introduce the function

$$
w=\tilde{K}+\left(1+x_{1}+\frac{w_{2}}{2 \beta}\right)^{\bar{\gamma}} .
$$

Now choose $\tilde{K}$ so large that, by Lemma 4.3 ,

$$
\bar{v}^{\theta}=(1-\theta) \bar{v}+\theta w, \quad \theta \in(0,1]
$$

is a strict supersolution of (4.1) in any bounded subset of $\mathcal{D}$. Instead of comparing $\underline{v}$ and $\bar{v}$, we will compare $\underline{v}$ and $\bar{v}^{\theta}$. Then by simply sending $\theta \rightarrow 0+$, we obtain the desired comparison result $\underline{v} \leq \bar{v}$ in $\overline{\mathcal{D}}$. Observe that

$$
\underline{v}(X)-\bar{v}^{\theta}(X) \leq \text { Const } \cdot\left(1+x_{1}+x_{2}\right)^{\gamma^{\prime}}-\theta\left(1+x_{1}+\frac{x_{2}}{2 \beta}\right)^{\bar{\gamma}} \rightarrow-\infty \text { as } X \rightarrow \infty .
$$


In view of $(4.12)$, we can choose $R>0$ so large that $\underline{v} \leq \bar{v}^{\theta}$ in $\left\{x_{1}, x_{2} \geq R\right\}$. Although $\mathcal{D}$ is unbounded, we can then nevertheless restrict our attention to the bounded domain

$$
\mathcal{K}=\left\{X=\left(x_{1}, x_{2}\right): 0<x_{1}<R+R e^{1}, 0<x_{2}<R\right\}
$$

and prove that $\underline{v} \leq \bar{v}^{\theta}$ in $\overline{\mathcal{K}}$. To this end, assume to the contrary that

$$
M:=\max _{\bar{\kappa}}\left(\underline{v}-\bar{v}^{\theta}\right)=\left(\underline{v}-\bar{v}^{\theta}\right)(Z)>0
$$

for some $Z \in \overline{\mathcal{K}}$. Observe that we have only the two cases $Z \in(0, R) \times(0, R)$ and $Z \in \Gamma_{\mathrm{SC}}$ to consider, where

$$
\Gamma_{\mathrm{SC}}=\left\{\left(x_{1}, x_{2}\right): x_{1}=0,0 \leq x_{2}<R \text { or } 0 \leq x_{1}<R, x_{2}=0\right\}
$$

is the state constraint boundary restricted by $R$.

Case I: Let us first consider the case $Z \in \Gamma_{\mathrm{SC}}$. The construction presented below is a suitable adaption of the construction of Soner $[33,34]$. Since $\partial \mathcal{K}$ is piecewise linear there exist constants $h_{0}, \kappa>0$ and a uniformly continuous map $\eta: \overline{\mathcal{K}} \rightarrow \mathbb{R}^{2}$ satisfying

$$
\mathcal{N}(X+h \eta(X), h \kappa) \subset \mathcal{K} \text { for all } X \in \overline{\mathcal{K}} \text { and } h \in\left(0, h_{0}\right]
$$

where $\mathcal{N}(z, \rho)$ denotes the ball with radius $\rho$ and centre $z$. For any $\alpha>1$ and $0<\varepsilon<1$, define the function $\Phi(X, Y)$ on $\overline{\mathcal{K}} \times \overline{\mathcal{K}}$ by

$$
\Phi(X, Y)=\underline{v}(X)-\bar{v}^{\theta}(Y) \cdots|\alpha(X-Y)+\varepsilon \eta(Z)|^{2}-\varepsilon|X-Z|^{2} .
$$

Let

$$
M_{\alpha}=\max _{\overline{\mathcal{K}} \times \overline{\mathcal{K}}} \Phi(X, Y) .
$$

We then have $M_{\alpha}>0$ for any $\alpha>1$ and $\varepsilon \leq \varepsilon_{0}$, where $\varepsilon_{0}$ is some fixed small number. Let $\left(X_{\alpha}, Y_{\alpha}\right) \in \overline{\mathcal{K}} \times \overline{\mathcal{K}}$ be a maximizer of $\Phi$, i.e., $M_{\alpha}=\Phi\left(X_{\alpha}, Y_{\alpha}\right)$. By (4.16), we assume that $\alpha$ is so large that $Z+\frac{\varepsilon}{\alpha} \eta(Z) \in \mathcal{K}$. The inequality

$$
\Phi\left(X_{\alpha}, Y_{\alpha}\right) \geq \Phi\left(Z, Z+\frac{\varepsilon}{\alpha} \eta(Z)\right)
$$

reads

$$
\begin{aligned}
& \left|\alpha\left(X_{\alpha}-Y_{\alpha}\right)+\varepsilon \eta(Z)\right|^{2}+\varepsilon\left|X_{\alpha}-Z\right|^{2} \\
& \quad \leq \underline{v}\left(X_{\alpha}\right)-\bar{v}^{\theta}\left(Y_{\alpha}\right)-\left(\underline{v}-\bar{v}^{\theta}\right)(Z)+\bar{v}^{\theta}\left(Z+\frac{\varepsilon}{\alpha} \eta(Z)\right)-\bar{v}^{\theta}(Z) .
\end{aligned}
$$

Since $\underline{v},-\bar{v}^{\theta}$ are bounded on $\overline{\mathcal{K}}$, it follows that $\left|\alpha\left(X_{\alpha}-Y_{\alpha}\right)\right|$ is bounded uniformly in $\alpha$. Hence $X_{\alpha}-\bar{Y}_{\alpha} \rightarrow 0$ as $\alpha \rightarrow \infty$ and $\lim _{\alpha \rightarrow \infty}\left(\underline{v}\left(X_{\alpha}\right)-\bar{v}^{\theta}\left(Y_{\alpha}\right)\right) \leq M$. Sending $\alpha \rightarrow \infty$ in (4.18) and using the uniform continuity of $\underline{v}, \bar{v}^{\theta}$ in $\overline{\mathcal{K}}$, we then conclude that $\alpha\left(X_{\alpha}-Y_{\alpha}\right)+\varepsilon \eta(Z) \rightarrow 0, X_{\alpha}, Y_{\alpha} \rightarrow Z$, and $M_{\alpha} \rightarrow M$. Therefore, using the uniform continuity of $\eta$,

$$
Y_{\alpha}=X_{\alpha}+\frac{\varepsilon}{\alpha} \eta(Z)+o\left(\frac{1}{\alpha}\right)=X_{\alpha}+\frac{\varepsilon}{\alpha} \eta\left(X_{\alpha}\right)+o\left(\frac{1}{\alpha}\right)
$$

and we thus use (4.16) to get $Y_{\alpha} \in \mathcal{K}$ for $\alpha$ large enough. In fact, since $Z \in \Gamma_{\mathrm{SC}}$, we must have

$$
X_{\alpha} \in[0, R) \times[0, R), \quad Y_{\alpha} \in(0, R) \times(0, R),
$$

for $\alpha$ large enough. Now define

$$
\begin{aligned}
& \psi(Y)=\underline{v}\left(X_{\alpha}\right)-\left|\alpha\left(X_{\alpha}-Y\right)+\varepsilon \eta(Z)\right|^{2}-\varepsilon\left|X_{\alpha}-Z\right|^{2}, \\
& \phi(X)=\bar{v}^{\theta}\left(Y_{\alpha}\right)+\left|\alpha\left(X-Y_{\alpha}\right)+\varepsilon \eta(Z)\right|^{2}+\varepsilon\left|X_{\alpha}-Z\right|^{2}
\end{aligned}
$$

and set

$$
\begin{aligned}
& P=D_{X} \phi\left(X_{\alpha}\right)=2 \alpha\left[\alpha\left(X_{\alpha}-Y_{\alpha}\right)+\varepsilon \eta(Z)\right]+2 \varepsilon\left(X_{\alpha}-Z\right), \\
& Q=D_{Y} \psi\left(Y_{\alpha}\right)=2 \alpha\left[\alpha\left(X_{\alpha}-Y_{\alpha}\right)+\varepsilon \eta(Z)\right] .
\end{aligned}
$$

Since $\bar{v}^{\theta}-\psi$ takes its minimum at $Y_{\alpha} \in \mathcal{K}$ and $\bar{v}^{\theta}$ is a strict supersolution in $\mathcal{K}, G(Q)<-\theta f$ and $F\left(Y_{\alpha}, \bar{v}^{\theta}, Q, \mathcal{B}^{\pi}\left(Y_{\alpha}, \psi\right)\right)<-\theta f$. Repeating the proof of Lemma 4.1, we see that the latter strict inequality implies

$$
F\left(Y_{\alpha}, \bar{v}^{\theta}, Q, \mathcal{B}^{\pi, \kappa}\left(Y_{\alpha}, \bar{v}^{\theta}, Q\right), \mathcal{B}_{k}^{\pi}\left(Y_{\alpha}, \psi\right)\right)<-\theta f
$$


We next claim that $G(P)<0$. Assume to the contrary that $G(P) \geq 0$. Then it follows that

$$
-\theta f>G(Q)-G(P)=\beta\left(q_{2}-p_{2}\right)-\left(q_{1}-p_{1}\right)=-2 \beta \varepsilon\left(x_{\alpha 2}-z_{2}\right)-2 \varepsilon\left(x_{\alpha 1}-z_{1}\right),
$$

which tends to zero as $\alpha \rightarrow \infty$, a contradiction to the fact that $f$ is strictly positive on $\mathcal{K}$. Thus our claim holds. Then since $\underline{v}-\phi$ takes its maximum at $X_{\alpha} \in \overline{\mathcal{K}}$ and $\underline{v}$ is a subsolution in $\overline{\mathcal{K}}$, $F\left(X_{\alpha}, \underline{v}, P, \mathcal{B}^{\pi}\left(X_{\alpha}, \phi\right)\right) \geq 0$. This in turn implies that

$$
F\left(X_{\alpha}, \underline{v}, P, \mathcal{B}^{\pi, \kappa}\left(X_{\alpha}, \underline{v}, P\right), \mathcal{B}_{\kappa}^{\pi}\left(X_{\alpha}, \phi\right)\right) \geq 0 \text {. }
$$

Using (4.20) and (4.21), we can calculate as follows

$$
\begin{aligned}
0<F & \left(X_{\alpha}, \underline{v}, P, \mathcal{B}^{\pi, \kappa}\left(X_{\alpha}, \underline{v}, P\right), \mathcal{B}_{k}^{\pi}\left(X_{\alpha}, \phi\right)\right)-F\left(Y_{\alpha}, \bar{v}^{\theta}, Q, \mathcal{B}^{\pi, \kappa}\left(Y_{\alpha}, \bar{v}^{\theta}, Q\right), \mathcal{B}_{\kappa}^{\pi}\left(Y_{\alpha}, \psi\right)\right) \\
\leq[ & \left.U\left(x_{\alpha 2}\right)-U\left(y_{\alpha 2}\right)\right]-\delta\left[\underline{v}\left(X_{\alpha}\right)-\bar{v}^{\theta}\left(Y_{\alpha}\right)\right]-\beta\left[x_{\alpha 2} \phi_{x_{2}}\left(X_{\alpha}\right)-y_{\alpha 2} \psi_{y_{2}}\left(Y_{\alpha}\right)\right] \\
& +\max _{\pi \in[0,1]}\left[(r+(\hat{\mu}-r) \pi)\left[x_{\alpha 1} \phi_{x_{1}}\left(X_{\alpha}\right)-y_{\alpha 1} \psi_{y_{1}}\left(Y_{\alpha}\right)\right]\right. \\
& \left.+\left[\mathcal{B}^{\pi, \kappa_{i}}\left(X_{\alpha}, \underline{v}, P\right)-\mathcal{B}^{\pi, \kappa}\left(Y_{\alpha}, \bar{v}^{\theta}, Q\right)\right]+\left[\mathcal{B}_{k}^{\pi}\left(X_{\alpha}, \phi\right)-\mathcal{B}_{r}^{\pi}\left(Y_{\alpha}, \psi\right)\right]\right] .
\end{aligned}
$$

Let us start by estimating the integral terms. To this end, observe first that, thanks to (4.2), $\mathcal{B}_{\kappa}^{\pi}\left(X_{\alpha}, \phi\right)$ and $\mathcal{B}_{k}^{\pi}\left(Y_{\alpha}, \psi\right)$ both tend to zero as $\kappa \rightarrow 0$ (for any finite $\alpha$ ). Next, for simplicity of presentation, introduce the short-hand notation $T^{\pi}(z ; X)=\left(x_{1}+\pi x_{1}\left(e^{z}-1\right), x_{2}\right)$ and note that (for fixed $X)\left|T^{\pi}(z ; X)\right|$ grows like $\left|e^{z}-1\right|$ as $z \rightarrow \infty$. Then

$$
\mathcal{B}^{\pi, \kappa}\left(X_{\alpha}, \underline{v}, P\right)-B^{\pi, \kappa}\left(Y_{\alpha}^{\prime}, \bar{v}^{\theta}, Q\right)=I_{1}+I_{2},
$$

where, for $A_{1}=\{\kappa<|z|<1\}$ and $A_{2}=\{|z| \geq 1\}$,

$$
\begin{aligned}
I_{\ell}=\int_{A_{\ell}}\left(\left[\underline{v}\left(T^{\pi}\left(z ; X_{\alpha}\right)\right)-\bar{v}^{\theta}\left(T^{\pi}\left(z ; Y_{\alpha}\right)\right)\right]-\left[\underline{v}\left(X_{\alpha}\right)-\bar{v}^{\theta}\left(Y_{\alpha}\right)\right]\right. \\
\left.-\pi\left[x_{\alpha 1} \phi_{2_{1}}\left(X_{\alpha}\right)-y_{\alpha 1} \psi_{y_{1}}\left(Y_{\alpha}\right)\right]\left(e^{z}-1\right)\right) \nu(d z), \quad \ell=1,2 .
\end{aligned}
$$

We consider first the term $I_{2}$. Observe that, for $i=1,2$,

$$
\begin{aligned}
& {\left[x_{\alpha i} \phi_{x_{i}}\left(X_{\alpha}\right)-y_{\alpha i} \psi_{y_{i}}\left(Y_{\alpha}\right)\right]} \\
& \left.\quad=\left(x_{\alpha i}-y_{\alpha i}\right) 2 \alpha\left[\alpha\left(x_{\alpha i}-y_{\alpha i}\right)+\varepsilon \eta_{i}(Z)\right]+2 \varepsilon x_{\alpha i}\left(x_{\alpha i}-z_{i}\right)\right]=\omega_{1}\left(\frac{1}{\alpha}\right),
\end{aligned}
$$

for some continuity modulus $\omega_{1}$. Since (4.13) and (4.14) obviously imply

$$
\left(\underline{v}-\bar{v}^{\theta}\right)(X) \leq M \text { for all } X \in \overline{\mathcal{D}}
$$

we get

$$
\begin{aligned}
I_{2} \leq \int_{|z| \geq 1}(M & +\bar{v}^{\theta}\left(T^{\pi}\left(z ; X_{\alpha}\right)\right)-\bar{v}^{\theta}\left(T^{\pi}\left(z ; Y_{\alpha}\right)\right)-M_{\alpha} \\
& \left.-\left[x_{\alpha 1} \phi_{x_{1}}\left(X_{\alpha}\right)-y_{\alpha 1} \psi_{y_{1}}\left(Y_{\alpha}\right)\right]\left(e^{z}-1\right)\right) \nu(d z) \\
\leq\left(M-M_{\alpha}\right) & \int_{|z| \geq 1} 1 \nu(d z)+\int_{|z| \geq 1}\left(\bar{v}^{\theta}\left(T^{\pi}\left(z ; X_{\alpha}\right)\right)-\bar{v}^{\theta}\left(T^{\pi}\left(z ; Y_{\alpha}\right)\right)\right) \nu(d z) \\
& +\omega_{1}\left(\frac{1}{\alpha}\right) \int_{|z| \geq 1}\left|e^{z}-1\right| \nu(d z) \rightarrow 0 \text { as } \alpha \rightarrow \infty,
\end{aligned}
$$

where we have exploited that $M_{\alpha} \rightarrow M$ as $\alpha \rightarrow \infty,(2.2)$, continuity of $\bar{v}^{\theta},(4.24)$, and (2.4)

We next estimate $I_{1}$. To this end, notice that (4.19) implies $T^{\pi}\left(z ; X_{\alpha}\right), T^{\pi}\left(z ; Y_{\alpha}\right) \in \mathcal{K}$ for $z \in(-1,1)$ and thus

$$
\Phi\left(T^{\pi}\left(z ; X_{\alpha}\right), T^{\pi}\left(z ; Y_{\alpha}\right)\right)-\Phi\left(X_{\alpha}, Y_{\alpha}\right) \leq 0
$$

A calculation reveals that the integrand of $I_{1}$ equals

$$
\Phi\left(T^{\pi}\left(z ; X_{\alpha}\right), T^{\pi}\left(z ; Y_{\alpha}\right)\right)-\Phi\left(X_{\alpha}, Y_{\alpha}\right)+\left(\pi^{2}\left[\alpha\left(x_{\alpha 1}-y_{\alpha 1}\right)\right]^{2}+\varepsilon \pi^{2} x_{\alpha 1}^{2}\right)\left(e^{z}-1\right)^{2}
$$


which, thanks to $(4.25)$, is less than or equal to

$$
\left(\left[\alpha\left(x_{\alpha 1}-y_{\alpha 1}\right)\right]^{2}+\varepsilon x_{\alpha 1}^{2}\right)\left(e^{z}-1\right)^{2} .
$$

Hence

$$
I_{1} \leq\left(\left[\dot{\alpha}\left(x_{\alpha 1}-y_{\alpha 1}\right)\right]^{2}+\varepsilon x_{\alpha 1}^{2}\right) \int_{\kappa<|z|<1}\left(e^{z}-1\right)^{2} \nu(d z) .
$$

Note that the integral is convergent since every Lévy measure integrates $z^{2}$ in a neighbourhood of zero, see (2.2). Since $\alpha\left(x_{\alpha 1}-y_{\alpha 1}\right) \rightarrow-\varepsilon \eta_{1}(Z)$ as $\alpha \rightarrow \infty$, we conclude that

$$
\lim _{\varepsilon \rightarrow 0} \lim _{\alpha \rightarrow \infty} I_{1} \leq 0 .
$$

Finally, in view of the estimates derived above, we send (in that order) $\alpha \rightarrow \infty, \varepsilon \rightarrow 0$, and $\kappa \rightarrow 0$ in (4.22) to obtain the desired contradiction

$$
\delta\left[\underline{v}(Z)-\bar{v}^{\theta}(Z)\right]<0 .
$$

Case II: Let us now consicler the case $Z \in(0, R) \times(0, R)$. For any $\alpha>1$ and $0<\varepsilon<1$, define the function $\Phi(X, Y)$ on $\overline{\mathcal{K}} \times \overline{\mathcal{K}}$ by

$$
\Phi(X, Y)=\underline{v}(X)-\bar{v}^{\theta}(Y)-\frac{\alpha}{2}|X-Y|^{2} .
$$

Let $M_{\alpha}=\max _{\overline{\mathcal{K}} \times \overline{\mathcal{K}}} \Phi(X, Y)$. We then have $M_{\alpha} \geq M>0$ for all $\alpha>1$. Let $\left(X_{\alpha}, Y_{\alpha}\right)$ be a maximizer so that $M_{\alpha}=\Phi\left(X_{\alpha}, Y_{\alpha}\right)$ and note that the inequality

$$
\Phi\left(X_{\alpha}, X_{\alpha}\right)+\Phi\left(Y_{\alpha}, Y_{\alpha}\right) \leq 2 \Phi\left(X_{\alpha}, Y_{\alpha}\right)
$$

implies

$$
\alpha\left|X_{\alpha}-Y_{\alpha}\right|^{2} \leq \underline{v}\left(X_{\alpha}\right)-\underline{v}\left(Y_{\alpha}\right)+\bar{v}^{\theta}\left(X_{\alpha}\right)-\bar{v}^{\theta}\left(Y_{\alpha}\right) .
$$

Consequently, we have the estimate $\left|X_{\alpha}-Y_{\alpha}\right| \leq K \sqrt{\frac{1}{\alpha}}$, where $K>0$ is a constant that depends on $\sup _{\mathcal{K}} \underline{v}$ and $\sup \overline{\mathcal{K}}\left(-\bar{v}^{\theta}\right)$. Hence, if $\left(X_{\alpha}, Y_{\alpha}\right) \rightarrow(\hat{X}, \hat{Y})$ as $\alpha \rightarrow \infty$, we have $\hat{X}=\hat{Y}$. Inserting the above estimate into (4.27) and using the uniform continuity of $\underline{v}, \bar{v}^{\theta}$ in $\overline{\mathcal{K}}$, we get $\alpha\left|X_{\alpha}-Y_{\alpha}\right|^{2} \rightarrow 0$ as $\alpha \rightarrow \infty$. Moreover, using $M \leq M_{\alpha}$, it then follows that

$$
0=\lim _{\alpha \rightarrow \infty} \frac{\alpha}{2}\left|X_{\alpha}-Y_{\alpha}\right|^{2} \leq \lim _{\alpha \rightarrow \infty}\left\{\underline{v}\left(X_{\alpha}\right)-\bar{v}^{\theta}\left(Y_{\alpha}\right)-M\right\}=\underline{v}(\hat{X})-\bar{v}^{\theta}(\hat{X})-M \leq 0,
$$

and we conclude that $M_{\alpha} \rightarrow M$ as $\alpha \rightarrow \infty$.

Since (4.14) holds and, thanks to Case I, $\underline{v} \leq \bar{v}^{\theta}$ on $\partial\{(0, R) \times(0, R)\}$, we conclude that any limit point of $\left(X_{\alpha}, Y_{\alpha}\right)$ belongs to $(0, R) \times(0, R)$. Hence for large enough $\alpha$,

$$
X_{\alpha}, Y_{\alpha} \in(0, R) \times(0, R) .
$$

Following the classical viscosity theory, let

$$
\psi(Y)=\underline{v}\left(X_{\alpha}\right)-\frac{\alpha}{2}\left|X_{\alpha}-Y\right|^{2}, \quad \phi(X)=\bar{v}^{\theta}\left(Y_{\alpha}\right)+\frac{\alpha}{2}\left|X-Y_{\alpha}\right|^{2} .
$$

Finally, set

$$
P=D_{X} \phi\left(X_{\alpha}\right)=\alpha\left(X_{\alpha}-Y_{\alpha}\right), \quad Q=D_{Y} \psi\left(Y_{\alpha}\right)=\alpha\left(X_{\alpha}-Y_{\alpha}\right) .
$$

Since $\bar{v}^{\theta}-\psi$ takes its minimum at $Y_{\alpha}$ and $\bar{v}^{\theta}$ is a strict supersolution, we have $G(Q)<-\theta f$ and $F\left(Y_{\alpha}, \bar{v}^{\theta}, Q, \mathcal{B}^{\pi}\left(Y_{\alpha}, \psi\right)<-\theta f\right.$, which also implies

$$
F\left(Y_{\alpha}, \bar{v}^{\theta}, Q, \mathcal{B}^{\pi, \kappa}\left(Y_{\alpha}, \bar{v}^{\theta}, Q\right), \mathcal{B}_{k i}^{\pi}\left(Y_{\alpha}, \psi\right)\right)<-\theta f .
$$

Assume that $G(P)>0$. Then it follows that $-\theta f>G(Q)-G(P) \equiv 0$, which is a contradiction. Thus, $G(P) \leq 0$. Now since $\underline{v}-\phi$ takes its maximum at $X_{\alpha}$ and $\underline{v}$ is a subsolution, $F\left(X_{\alpha}, \underline{v}, P, B \phi\right) \geq 0$, which also implies

$$
F\left(X_{\alpha}, \underline{v}, P, \mathcal{B}^{\pi, \kappa}\left(X_{\alpha}, \underline{v}, P\right), \mathcal{B}_{\kappa}^{\pi}\left(X_{\alpha}, \phi\right)\right) \geq 0 .
$$


Using (4.29) and (4.30), we get (consult Case I)

$$
\begin{aligned}
& 0<F\left(X_{\alpha}, \underline{v}, P, \mathcal{B}^{\pi, \kappa}\left(X_{\alpha}, \underline{v}, P\right), \mathcal{B}_{\kappa}^{\pi}\left(X_{\alpha}, \phi\right)\right)-F\left(Y_{\alpha}, \bar{v}^{\theta}, Q, \mathcal{B}^{\pi, k}\left(Y_{\alpha}, \bar{v}^{\theta}, Q\right), \mathcal{B}_{k}^{\pi}\left(Y_{\alpha}, \psi\right)\right) \\
& \leq\left[U\left(x_{\alpha 2}\right)-U\left(y_{\alpha 2}\right)\right]-\delta\left[\underline{v}\left(X_{\alpha}\right)-\bar{v}^{\theta}\left(Y_{\alpha}\right)\right]-\beta\left[x_{\alpha 2} \phi_{v_{2}}\left(X_{\alpha}\right)-y_{\alpha 2} \psi_{y_{2}}\left(Y_{\alpha}\right)\right] \\
& \quad \quad \quad \max _{\pi \in[0,1]}\left[(r+(\mu-r) \pi)\left[x_{\alpha 1} \phi_{x_{1}}\left(X_{\alpha}\right)-y_{\alpha 1} \psi_{y_{1}}\left(Y_{\alpha}\right)\right]+I_{1}+I_{2}+\left[\mathcal{B}_{k}^{\pi}\left(X_{\alpha}, \phi\right)-\mathcal{B}_{*}^{\pi}\left(Y_{\alpha}, \psi\right)\right]\right],
\end{aligned}
$$

where $I_{1}, I_{2}$ are defined in (4.23) with $\phi, \psi$ defined in (4.28).

Observe that in the present case, (4.24) reads

$$
\left[x_{\alpha i} \phi_{x_{i}}\left(X_{\alpha}\right)-y_{\alpha i} \psi_{y_{i}}\left(Y_{\alpha}\right)\right]=\alpha\left(x_{\alpha i}-y_{\alpha i}\right)^{2}=\omega_{3}\left(\frac{1}{\alpha}\right), \quad i=1,2,
$$

for some continuity modulus $\omega_{3}$. Appealing once more to $(4.2)$, we know that $\mathcal{B}_{k}^{\pi}\left(X_{\alpha}, \phi\right)$ and $\mathcal{B}_{\kappa}^{\pi}\left(Y_{\alpha}, \psi\right)$ tend to zero as $\kappa \rightarrow 0$. Moreover, $\lim _{\alpha \rightarrow \infty} I_{2} \leq 0$ (consult Case $\mathrm{I}$ ). To estimate the integral $I_{1}$, we note that the integrand equals

$$
\Phi\left(T^{\pi}\left(z ; X_{\alpha}\right), T^{\pi}\left(z ; Y_{\alpha}\right)\right)-\Phi\left(X_{\alpha}, Y_{\alpha}\right)+\pi^{2} \frac{\alpha}{2}\left(x_{\alpha 1}-y_{\alpha 1}\right)^{2}\left(e^{z}-1\right)^{2}
$$

Obviously, $T^{\pi}\left(z ; X_{\alpha}\right), T^{\pi}\left(z ; Y_{\alpha}\right) \in \overline{\mathcal{K}}$ and thus

$$
\Phi\left(T^{\pi}\left(z ; X_{\alpha}\right), T^{\pi}\left(z ; Y_{\alpha}\right)\right)-\Phi\left(X_{\alpha}, Y_{\alpha}\right) \leq 0
$$

Since $\alpha\left|X_{\alpha}-Y_{\alpha}\right|^{2} \rightarrow 0$ as $\alpha \rightarrow \infty$, we obtain

$$
I_{1} \leq \frac{\alpha}{2}\left(x_{\alpha 1}-y_{\alpha 1}\right)^{2} \int_{\kappa<|z|<1}\left(e^{z}-1\right)^{2} \nu(d z) \rightarrow 0 \text { as } \alpha \rightarrow \infty .
$$

Sending (in that order) $\alpha \rightarrow \infty$ and $\kappa \rightarrow 0$ in (4.31), we obtain as in Case I the contradiction (4.26). This concludes the proof of the theorem.

Remark 4.1. We remark that Theorem 4.2 also holds for a class of second order integ'ro-differential variational inequalities, see [7] for details.

\section{REFERENCES}

[1] O. Alvarez, A singular stochastic control problem in an unbounded domain, Comm. Partial Differential Equations 19 (1994), no. 11-12, 2075-2089.

[2] O. Alvarez and A. Tourin, Viscosity solutions of nonlinear integro-differential equations, Ann. Inst. H. Poincaré Anal. Non Linéaire 13 (1996), no. 3, 293-317.

[3] M. Bardi and I. Capuzzo-Dolcetta, Optimal control and viscosity solutions of Hamilton-Jacobi-Bellman equations. Birkhäuser Boston, Boston, MA, 1997.

[4] M. Bardi et al,, Viscosity solutions and applications, Lecture Notes in Math., 1660, Springer, Berlin, 1997.

[5] G. Barles, Solutions de viscosité des équations de Hamilton-Jacobi, Springer, Paris, 1994.

[6] O. E. Barndorff-Nielsen, Processes of Normal inverse Gaussian type, Finance and Stochastics 2 (1998), 41-68.

[7] F. E. Benth, K. H. Karlsen, and K. Reikvam, Optimal portfolio management rules in a non-Gaussian market with durability and intertemporal substitution. Preprint, Department of Mathematics, University of Bergen, 2000.

[8] M. G. Cranclall, Viscosity solutions: a primer, in Viscosity solutions and applications (Montecatini Terme, 1995), 1-43, Lecture Notes in Math., 1660, Springer, Berlin, 1997.

[9] M. G. Crandall, L. C. Evans, and P.-L. Lions, Some properties of viscosity solutions of Hamilton-Jacobi equations, Trans. Amer. Math. Soc, 282 (1984), no. 2, 487-502.

[10] M. G. Crandall, H. Ishii, and P.-L. Lions, User's guide to viscosity solutions of second order partial differential equations, Bull. Amer. Math. Soc. (N.S.) 27 (1992), no, 1, 1-67.

[11] M. G. Crandall and P.-L. Lions, Viscosity solutions of Hamilton-Jacobi equations, Trans. Amer. Math. Soc. 277 (1983), no. $1,1-42$

[12] M. H. A. Davis, V. G. Panas, and T. Zariphopoulou, European option pricing with transaction costs, SIAM J. Control Optim. 31 (1993), no. 2, 470-493.

[13] I. Capuzzo-Dolcetta and P.-L. Lions, Hamilton-Jacobi equations with state constraints, Trans. Amer. Math. Soc. 318 (1990), no. 2, 643-683.

[14] E. Eberlein and U. Keller, Hyperbolic distributions in finance, Bernoulli 1(3) (1995), 281-299.

[15] W. H. Fleming and H. M. Soner, Controlled Martiov Processes and Viscosity Solutions, Applications of Mathematics 25, New York: Springer Verlag, (1993).

[16] A. Hindy and C. Huang, Optimal consumption and portfolio rules with durability and local substitution, Econometrica 61 (1993), 85-121. 
[17] A. Hindy, C. Huang, and H. Zhu, Numerical analysis of a free-boundary singular control problem in financial economics, J. of Economics Dynamics and Control 21 (1997), 297-327.

[18] N. Ikeda and S. Watanabe, Stochastic Differential Equations and Diffusion Processes, 2nd Edition, NorthHolland/Kodansha, (1989).

[19] H. Ishii, On uniqueness and existence of viscosity solutions of fully nonlinear seconcl-order elliptic PDEs, Comm. Pure Appl. Math. 42 (1989), no. 1, 15-45.

[20] H. Ishii and P.-L. Lions, Viscosity solutions of fully nonlinear second-order elliptic partial differential equations, J. Differential Equations 83 (1990), no. 1, 26-78.

[21] R. Jensen, The maximum principle for viscosity solutions of fully nonlinear second order partial differential equations, Arch. Rational Mech. Anal. 101 (1988), no. 1, 1-27.

[22] R. Jensen, Uniqueness criteria for viscosity solutions of fully nonlinear elliptic partial differential equations, Indiana Univ. Math. J. 38 (1989), no. 3, 629-667.

[23] M. A. Katsoulakis, Viscosity solutions of second order fully nonlinear elliptic equations with state constraints, Indiana Univ. Math. J, 43 (1994), no. 2, 493-519.

[24] J.-M. Lasry and P.-L. Lions, Nonlinear elliptic equations with singular boundary conditions and stochastic control with state constraints. I. The model problem, Math. Ann. 283 (1989), no. 4, 583-630.

[25] R. Jensen, P.-L. Lions, and P. E. Souganidis, A uniqueness result for viscosity solutions of second order fully nonlinear partial differential equations, Proc. Amer. Math. Soc. 102 (1988), no. 4, 975-978.

[26] P. L. Lions, Optimal control of diffusion processes and Hamilton-Jacobi-Bellman equations. I. The dynamic programming principle and applications, Comm. Partial Differential Equations 8 (1983), no. 10, 1101-1174.

[27] P. L. Lions, Optimal control of diffusion processes and Hamilton-Jacobi-Bellman equations. II. Viscosity solutions and uniqueness, Comm. Partial Differential Equations 8 (1983), no. 11, 1229-1276.

[28] P.-L. Lions and P. E. Souganidis, Viscosity solutions of second-order equations, stochastic control and stochastic differential games, in Stochastic differential systems, stochastic control theory and applications (Minneapolis, Minn., 1986), 293-309, Springer, New York, 1988.

[29] H. Pham, Optimal stopping of controlled jump diffusion processes: A viscosity solution approach. J. Math. Syst. Estim. Control 8 (1998), No,1, 27 pp.

[30] T. H. Rydberg, The normal inver'se Gaussian Lévy process: Simulation and approximation. Commun. Statist,Stochastic Models 13 (4) (1997), 887-910.

[31] A. Sayah, Équations d'Hamilton-Jacobi du premier ordre avec termes intégro-différentiels. I. Unicité des solutions de viscosité, Comm. Partial Differential Equations 16 (1991), no. 6-7, 1057-1074.

[32] A. Sayah, Équations d'Hamilton-Jacobi du premier ordre avec termes intégro-différentiels. II. Existence de solutions de viscosité, Comm. Partial Differential Equations 16 (1991), no. 6-7, 1075-1093.

[33] H. M. Soner, Optimal control with state-space constraint. I, SIAM J. Control Optim. 24 (1986), no. 3, 552-561.

[34] H. M. Soner, Optimal control with state-space constraint. II, SIAM J. Control Optim. 24 (1986), no. 6, $1110-1122$,

[35] H. M. Soner, Optimal control of jump-Markov processes and viscosity solutions, in Stochastic differential systems, stochastic control theory and applications (Minneapolis, Minn., 1986), IMA Vol. Math. Appl., 10, 501-511, Springer, New York, 1988.

[36] H. M. Soner, Controlled Markov processes, viscosity solutions and applications to mathematical finance, in Viscosity solutions and applications (Montecatini Terme, 1995), 134-185, Lecture Notes in Math., 1660, Springer, Berlin, 1997.

[37] S. Zhu, Dynamic programming and variational inequalities in singular stochastic control, Doctoral Dissertation, Division of Applied Mathematics, Brown University (1991). 
(Fred Espen Benth)

Department of Mathematics

UNIVERSITY OF OSLO

P.O. BoX 1053, BLINDERN

N-0316 OSLO, NORWAY

AND

MaPhySto - Centre for Mathematical Physics and Stochastics

UNIVERSITY OF AARHUS

NY MUNKEGADE

DK-8000 ARHus, DENMARK

E-mail address: fredb@math,uio,no

(Kenneth Hvistendahl Karlsen)

Department of Mathematics

UNIVERSITY OF BERGEN

JOHS, BRUNSGT. 12

N-5008 BERGEN, NORWAY

E-mail address: kenneth.karlsen@ni.uib.no

(Kristin Reikvam)

DEPARTMENT OF Mathematics

UNIVERSITY OF OSLO

P.O. BOX 1053, BLINDERN

N-0316 OSLO, NORWAY

E-mail address: kre@math.uio.no 